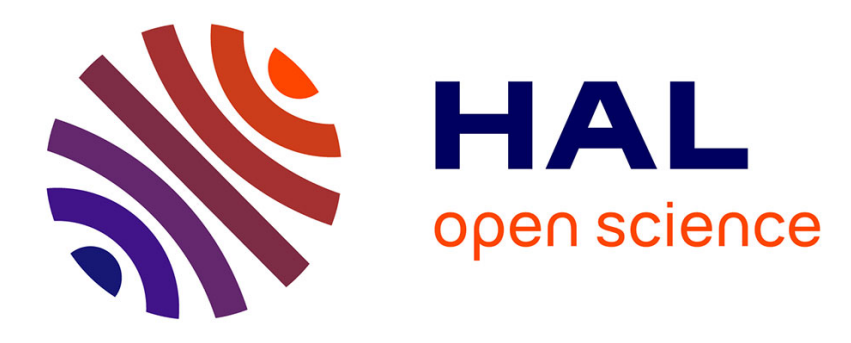

\title{
Dynamic geometric graph processes: adjacency operator approach
}

\author{
René Schott, Stacey Staples
}

\section{To cite this version:}

René Schott, Stacey Staples. Dynamic geometric graph processes: adjacency operator approach. Advances in Applied Clifford Algebras, 2010, 20 (3-4), pp.893-921. 10.1007/s00006-010-0228-6 . hal00438175

\section{HAL Id: hal-00438175 \\ https://hal.science/hal-00438175}

Submitted on 2 Dec 2009

HAL is a multi-disciplinary open access archive for the deposit and dissemination of scientific research documents, whether they are published or not. The documents may come from teaching and research institutions in France or abroad, or from public or private research centers.
L'archive ouverte pluridisciplinaire HAL, est destinée au dépôt et à la diffusion de documents scientifiques de niveau recherche, publiés ou non, émanant des établissements d'enseignement et de recherche français ou étrangers, des laboratoires publics ou privés. 


\title{
Dynamic Geometric Graph Processes: Adjacency Operator Approach
}

\author{
René Schott and G. Stacey Staples
}

\begin{abstract}
The $d$-dimensional unit cube $[0,1]^{d}$ is discretized to create a collection $V$ of vertices used to define geometric graphs. Each subset of $V$ is uniquely associated with a geometric graph. Defining a dynamic random walk on the subsets of $V$ induces a walk on the collection of geometric graphs in the discretized cube. These walks naturally model addition-deletion networks and can be visualized as walks on hypercubes with loops. Adjacency operators are constructed using subalgebras of Clifford algebras and are used to recover information about the cycle structure and connected components of the $n$ graph of a sequence.
\end{abstract}

\section{Introduction}

Consider $n$ points distributed uniformly and independently in the unit cube $[0,1]^{d}$. Given fixed real number $r>0$, connect two points by an edge if their Euclidean distance is at most $r$. Graphs of this type are called random geometric graphs, commonly denoted $G^{(d)}\left(\chi_{n}, ; r\right)[9]$.

Random geometric graphs are of particular interest as models of wireless networks [3], [6], [7], [1]. The vertices (or nodes) of the graph typically represent wireless devices that can communicate with each other when their physical distance is less than some prescribed range. Of particular interest is the graph's connectivity. Ad hoc networks are modeled by addition-deletion processes.

Considering a geometric graph on $n$ vertices, Xue and Kumar [20] have recently shown that the number of neighbors of each vertex needs to grow like $\Theta(\log n)$ if the graph is to be connected.

The philosophy presented in this paper is to first discretize the unit cube by partitioning it into sub-cubes whose center points serve as the vertices of a geometric graph. For fixed radius $r$, graphs are then uniquely determined by their vertex sets. A geometric graph process is then associated with a random walk on a hypercube of appropriate dimension induced by an algebraic process [17]. Other 
algebraic methods (cf. [12], [14], [16], [17]) are then applied to determine the cycle structure and connectivity properties of the graph on the discretized cube.

\section{Notational Preliminaries}

A graph $G=(V, E)$ is a collection of vertices $V$ and a set $E$ of unordered pairs of vertices called edges. Two vertices $v_{i}, v_{j} \in V$ are adjacent if there exists an edge $\left\{v_{i}, v_{j}\right\} \in E$. Given a graph $G$, it will sometimes be convenient to use the notation $V_{G}$ and $E_{G}$ to denote, respectively, the vertices and the edges of $G$.

A $k$-walk $\left(v_{0}, \ldots, v_{k}\right)$ in a graph $G$ is a sequence of vertices in $G$ with initial vertex $v_{0}$ and terminal vertex $v_{k}$ such that there exists an edge $\left\{v_{j}, v_{j+1}\right\} \in E$ for each $0 \leq j \leq k-1$. Note that a $k$-walk contains $k$ edges. A $k$-path is a $k$-walk in which no vertex appears more than once. A closed $k$-walk is a $k$-walk whose initial vertex is also its terminal vertex. A $k$-cycle $(k \geq 3)$ is a closed $k$-path with $v_{0}=v_{k}$. A Hamiltonian cycle is an $n$-cycle in a graph on $n$ vertices; i.e., it contains $V$. An edge from a vertex to itself is called a loop.

The circumference of a graph is the length of the longest cycle contained in the graph. The girth of a graph is defined as the length of the shortest cycle contained in the graph.

A graph $G$ is said to be connected if for every pair $u \neq v \in V_{G}$, there exists a $k$-walk from $u$ to $v$ for some positive integer $k$. A component of $G$ is a connected subgraph of maximal size contained in $G$.

A tree is a connected graph that contains no $k$-cycles for $k \geq 3$. A spanning tree in a graph $G$ is a subgraph of $G$ that forms a tree and contains all vertices of $G$.

\subsection{Random geometric graphs}

A random graph $G(n, p)$ is a graph with $n$ vertices in which each possible edge is independently included with probability $p$.

Let $r$ be an arbitrary positive real number, and let $V=v_{1}, \ldots, v_{n}$ be a set of points in a metric space with norm $\|\cdot\|$. A geometric graph $G(V, r)$ is defined as the graph with vertex set $V$ and edge set $E$ defined by

$$
\left\{v_{i}, v_{j}\right\} \in E \Leftrightarrow 0<\left\|v_{i}-v_{j}\right\|<r .
$$

Definition 2.1. A random geometric graph $G(n, r)$ is a geometric graph in which the $n$ vertices are independently and uniformly distributed in a metric space. It is a random graph in which the edge existence probability $p$ between two vertices is defined by

$$
p= \begin{cases}1 & \text { if } 0<\left\|v_{i}-v_{j}\right\| \leq r, \\ 0 & \text { otherwise. }\end{cases}
$$

Consider first the unit $d$-cube $[0,1]^{d}$. Dividing the sides into $N$ equal subintervals yields $N^{d}$ sub-cubes. Center points of the sub $d$-cubes will serve as vertices of a geometric graph. 
The set of vertices $V$ is defined by

$$
V=\left\{\left(\frac{2 j_{1}-1}{2 N}, \ldots, \frac{2 j_{d}-1}{2 N}\right): 1 \leq j_{1}, \ldots, j_{d} \leq N\right\} .
$$

The partitioned $d$-cube just described will be said to have mesh $1 / N^{d}$.

Given any subset $U \subseteq V$, the topology of the geometric graph on vertex set $U$ is uniquely determined by

$$
v_{1} \sim v_{2} \Leftrightarrow 0<\left\|v_{1}-v_{2}\right\| \leq r .
$$

The geometric graph with vertex set $U$ will be denoted by $G_{U}$.

\subsection{Three useful algebras from Clifford algebras}

Given a collection of commuting null-square elements $\left\{\zeta_{j}\right\}$ in one-to-one correspondence with the vertex set $V$, let $\mathcal{C} \ell_{V}{ }^{\text {nil }}$ denote the associative algebra generated by $\left\{\zeta_{j}\right\}$ and the unit scalar $1=\zeta_{\emptyset}$. In particular, $\zeta_{i} \zeta_{j}=\zeta_{j} \zeta_{i}$ when $i \neq j$ and $\zeta_{i}{ }^{2}=0$ for each $i$.

For convenience, generators of $\mathcal{C} \ell_{V}{ }^{\text {nil }}$ will be labeled with elements of $V$. The basis of $\mathcal{C} \ell_{V}$ nil is then in one-to-one correspondence with the power set of $V$. For any subset $U \subseteq V$, define the blade $\zeta_{U}=\prod_{v \in U} \zeta_{v}$. An arbitrary element $z \in \mathcal{C} \ell_{V}$ nil then has canonical expansion of the form

$$
z=\sum_{U \subseteq V} \alpha_{U} \zeta_{U}
$$

where $\alpha_{U} \in \mathbb{R}$.

By convention a blade will refer to any basis monomial in an algebra.

The algebra $\mathcal{C} \ell_{V}{ }^{\text {nil }}$ is constructed within $\mathcal{C} \ell_{2|V|, 2|V|}$ as follows: define $f_{i}=$ $\left(\mathbf{e}_{i}-\mathbf{e}_{n+i}\right) \in \mathcal{C} \ell_{2|V|, 2|V|}$ for each $1 \leq i \leq 2|V|$. Then letting $\zeta_{i}=f_{2 i-1} f_{2 i}$ for $1 \leq i \leq|V|$ completes the construction.

Remark 2.2. The algebra $\mathcal{C} \ell_{V}{ }^{\text {nil }}$ is referred to as a zeon algebra by Feinsilver [2]. It is the algebra referred to as $\mathcal{N}_{V}$ in Schott and Staples [12].

Assuming a fixed enumeration of elements of $V$, a probability mapping $\varphi$ is induced on the generators of $\mathcal{C} \ell_{V}{ }^{\text {nil }}$ by

$$
\varphi\left(\zeta_{v_{j}}\right)=\mu\left(v_{j}\right)
$$

Denote by $\left\{e_{i}\right\}$ the collection of orthonormal basis vectors of $\mathbb{R}^{|V| 2^{|V|}}$. The Dirac notation $\left\langle e_{i}\right|$ will represent a row vector, while the conjugate transpose, $\left|e_{i}\right\rangle$ represents a column vector. In this way,

$$
\left\langle e_{i} \mid e_{j}\right\rangle= \begin{cases}1 & \text { if } i=j \\ 0 & \text { otherwise }\end{cases}
$$

Moreover, $\left|e_{i}\right\rangle\left\langle e_{i}\right|$ is the rank-one orthogonal projector onto the linear subspace $\operatorname{span}\left(e_{i}\right)$. 
Fix an enumeration $f: 2^{V} \rightarrow\left\{1, \ldots, 2^{|V|}\right\}$ of the power set $2^{V}$. Notation of the form $\left|e_{U}\right\rangle$ and $\left\langle e_{U}\right|$ should be understood to use the fixed enumeration of $2^{V}$ for subsets $U \subseteq V$.

Define an enumeration of $2^{V} \times V$ by

$$
\left(U,\left\{v_{j}\right\}\right) \mapsto(f(U)-1)|V|+j .
$$

The enumeration of $2^{V} \times V$ is then used as a double-index for the unit basis vectors of $\mathbb{R}^{|V| 2^{|V|}}$. Notation of the form $\left|e_{U, v_{i}}\right\rangle$ and $\left\langle e_{U, e_{i}}\right|$ should be viewed in this context.

For each subset of vertices $U \subseteq V$, denote the nilpotent adjacency operator of the corresponding subgraph $G_{U}$ by $\Phi_{r}^{(U)}$. In particular,

$$
\Phi_{r}^{(U)}=\sum_{\substack{v_{i}, v_{j} \in U \\ 0<\left\|v_{1}-v_{2}\right\|<N r}} \zeta_{\left\{v_{2}\right\}}\left|e_{U, v_{1}}\right\rangle\left\langle e_{U, v_{2}}\right| .
$$

Given a collection of commuting idempotent elements $\left\{\gamma_{j}\right\}$ in one-to-one correspondence with the vertex set $V$, let $\mathcal{C} \ell_{V}$ idem denote the associative algebra generated by $\left\{\gamma_{j}\right\}$ and the unit scalar $1=\gamma_{\emptyset}$. In particular, $\gamma_{i} \gamma_{j}=\gamma_{j} \gamma_{i}$ when $i \neq j$, and $\gamma_{i}^{2}=\gamma_{i}$ for each $i$.

The algebra $\mathcal{C} \ell_{V}{ }^{\text {idem }}$ is constructed within the Clifford algebra $\mathcal{C} \ell_{|V|,|V|}$ by letting $\gamma_{i}=\frac{1}{2}\left(1+\mathbf{e}_{i} \mathbf{e}_{|V|+i}\right) \in \mathcal{C} \ell_{|V|,|V|}$ for each $1 \leq i \leq|V|$.

For convenience, generators of $\mathcal{C} \ell_{V}$ idem will be labeled with elements of $V$. The basis of $\mathcal{C} \ell_{V}{ }^{\text {idem }}$ is then in one-to-one correspondence with the power set of $V$. For any subset $U \subseteq V$, define the notation $\gamma_{U}=\prod_{v \in U} \gamma_{v}$. An arbitrary element $z \in \mathcal{C} \ell_{V}{ }^{\text {idem }}$ then has canonical expansion of the form

$$
z=\sum_{U \subseteq V} \alpha_{U} \gamma_{U}
$$

where $\alpha_{U} \in \mathbb{R}$.

Define the degree mapping $\delta: \mathcal{C} \ell_{V}{ }^{\text {idem }} \rightarrow \mathbb{N}_{0}$ by

$$
\delta\left(\sum_{U \in 2^{V}} \alpha_{U} \gamma_{U}\right)=\max _{\alpha_{U} \neq 0}\{|U|\} .
$$

In other word, $\delta(z)$ is the size of the maximal multi-index in the canonical expansion of $z \in \mathcal{C} \ell_{V}$ idem.

For each subset $U$ of the collection of vertices $V$, denote the corresponding idempotent adjacency operator by

$$
\Xi_{r}^{(U)}=\sum_{\substack{v_{1}, v_{2} \in U \\\left\|v_{1}-v_{2}\right\| \leq r}} \gamma_{v_{2}}\left|e_{U, v_{1}}\right\rangle\left\langle e_{U, v_{2}}\right|
$$



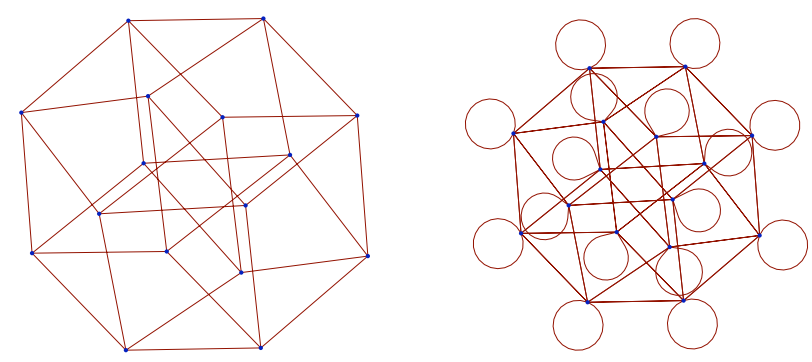

FiguRE 1. Graphs $\mathcal{Q}_{4}$ and $\tilde{\mathcal{Q}}_{4}$ associated with walk $\left(\Upsilon_{n}\right)$.

Remark 2.3. By using the inequality $\left\|v_{1}-v_{2}\right\| \leq r$ in place of $0<\left\|v_{1}-v_{2}\right\| \leq r$, "loops" are placed at each vertex of the graph. This allows every pair of vertices in a given component to be joined by a closed walk of length $2|V|-1$.

Given a collection of commuting unipotent elements $\left\{\varsigma_{j}\right\}$ in one-to-one correspondence with the vertex set $V$, let $\mathcal{C} \ell_{V}{ }^{\text {sym }}$ denote the associative algebra generated by $\left\{\varsigma_{j}\right\}$ and the unit scalar $1=\varsigma_{\emptyset}$. In particular, $\varsigma_{i} \varsigma_{j}=\varsigma_{j} \varsigma_{i}$ when $i \neq j$, and $\varsigma_{i}{ }^{2}=1$ for each $i$.

The algebra $\mathcal{C} \ell_{V}{ }^{\text {sym }}$ is constructed within the Clifford algebra $\mathcal{C} \ell_{|V|,|V|}$ by letting $\varsigma_{i}=\mathbf{e}_{i} \mathbf{e}_{|V|+i} \in \mathcal{C} \ell_{|V|,|V|}$ for each $1 \leq i \leq|V|$.

For convenience, generators of $\mathcal{C} \ell_{V}{ }^{\text {sym }}$ will be labeled with elements of $V$. The basis of $\mathcal{C} \ell_{V}{ }^{\text {sym }}$ is then in one-to-one correspondence with the power set of $V$. For any subset $U \subseteq V$, define the notation $\varsigma_{U}=\prod_{v \in U} \varsigma_{\{v\}}$. An arbitrary element $z \in \mathcal{C} \ell_{V}{ }^{\text {sym }}$ then has canonical expansion of the form

$$
z=\sum_{U \subseteq V} \alpha_{U} \varsigma_{U}
$$

where $\alpha_{U} \in \mathbb{R}$.

Note that each blade $\varsigma_{U}$ in $\mathcal{C} \ell_{V}{ }^{\text {sym }}$ is associated with a unique geometric graph $G_{U}$.

Each vertex of the hypercube $\mathcal{Q}_{|V|}$ is uniquely identified with a geometric graph. Hence, any graph process corresponds to a random walk on the hypercube $\mathcal{Q}_{|V|}$ or a hypercube with loops at each vertex $\tilde{\mathcal{Q}}_{|V|}$, depending on whether or not the walk is allowed to revisit the same graph at consecutive steps. 


\section{Graph Processes}

A geometric graph process is a sequence of random geometric graphs $\left(\mathcal{G}_{n}\right)_{n \geq 0}$ such that $\left|V_{n-1} \triangle V_{n}\right| \leq 1$ for each $n \geq 1$. Here, $\triangle$ is the set symmetric difference operator. An example is the sequence in Figure 3.

The strategy is now to define "second quantization" adjacency operators that encode information about the graphs in a graph process.

Use the fixed enumeration of $2^{V}$ to define the second quantization nilpotent adjacency operator by

$$
\Phi_{r}=\sum_{U \in 2^{V}}\left(\Phi_{r}^{(U)} \otimes\left|e_{U}\right\rangle\left\langle e_{U}\right|\right) .
$$

By construction, $\Phi_{r}$ is an operator on the $N^{d} 2^{N^{d}}$-dimensional product space $\mathcal{C} \ell_{V}{ }^{\text {nil }|V| 2^{|V|}}$. In particular, $\Phi_{r}$ is defined by

$$
\left\langle e_{U, v_{i}}\left|\Phi_{r}\right| e_{U, v_{j}}\right\rangle= \begin{cases}\zeta_{v_{j}} & \text { if } v_{i} \sim v_{j} \text { in } G_{U} \\ 0 & \text { otherwise }\end{cases}
$$

Recalling the canonical expansion $x=\sum_{U \subseteq V} x_{U} \zeta_{U} \in \mathcal{C} \ell_{V}{ }^{\text {nil }}$, let $\mathbb{N}_{0}=\{0,1,2, \ldots\}$ and define the function $\psi: \mathcal{C} \ell_{V}{ }^{\text {nil }} \rightarrow \mathbb{N}_{0}$ by

$$
\psi(x)=\sum_{U \subseteq V}\left\langle x, \zeta_{U}\right\rangle=\sum_{U \subseteq V} x_{u} .
$$

In other words, $\psi(x)$ is the sum of the scalar coefficients in the canonical expansion of $x$.

For convenience, define the notation $\vec{e}_{V}=e_{1}+e_{2}+\cdots+e_{|V| !}$, and for any $U \subseteq V$, define the $U$-trace of $\Phi_{r}$ by

$$
\operatorname{Tr}_{U}\left(\Phi_{r}\right)=\sum_{j=1}^{|V|}\left\langle e_{U, j}\left|\Phi_{r}\right| e_{U, j}\right\rangle
$$

The second quantization idempotent adjacency operator is defined by

$$
\Xi_{r}=\sum_{U \in 2^{V}}\left(\Xi_{r}^{(U)} \otimes\left|e_{U}\right\rangle\left\langle e_{U}\right|\right) .
$$

By construction, $\Xi_{r}$ is an operator on the $N^{d} 2^{N^{d}}$-dimensional product space $\mathcal{C} \ell_{V}{ }^{\text {idem }|V| 2^{|V|}}$. In particular, $\Xi_{r}$ is defined by

$$
\left\langle e_{U, v_{i}}\left|\Xi_{r}\right| e_{U, v_{j}}\right\rangle= \begin{cases}\gamma_{v_{j}} & \text { if } v_{i} \sim v_{j} \text { in } G_{U} \\ 0 & \text { otherwise. }\end{cases}
$$

It should be clear that because a geometric graph is uniquely determined by its vertex set, every graph process $\left(\mathcal{G}_{n}\right)$ is uniquely determined by a sequence $\left(U_{n}\right)$ of subsets of $V$ satisfying $\left|U_{n}\right|=n$ for each $n \geq 1$. Moreover, a graph process on 
the partitioned unit cube of mesh $1 / N^{d}$ is a random walk on a subgraph of the $N^{d}$-dimensional hypercube $\mathcal{Q}_{|V|}$ restricted to strictly increasing vertex weights.

Given a finite set $V$, vertices of the $|V|$-dimensional hypercube $\mathcal{Q}_{|V|}$ are represented by binary strings of length $|V|$. A one-to-one correspondence between the blades of $\mathcal{C} \ell_{V}{ }^{\text {sym }}$ and vertices of $\mathcal{Q}|V|$ is obtained through the binary string representation of subsets of $V$. Representing a binary string by $\left(b_{|V|} \ldots b_{2} b_{1}\right)$, where each $b_{i}$ takes values in $\{0,1\}$, such a correspondence takes the form

$$
\left(b_{|V|} \ldots b_{2} b_{1}\right) \mapsto \prod_{i: b_{i}=1} \varsigma_{\left\{v_{i}\right\}}
$$

For example, the blade representation of vertex (0101) in the hypercube $\mathcal{Q}_{4}$ is $\varsigma_{\left\{v_{1}, v_{3}\right\}} \in \mathcal{C} \ell_{V}$ sym.

To construct a random walk on the $|V|$-dimensional hypercube, one can think of a binary string of length $|V|$ and consider the effect of "flipping" a single digit, either from 0 to 1 or vice-versa, at each discrete time step. This can be accomplished within $\mathcal{C} \ell_{V}{ }^{\text {sym }}$ simply by multiplying the blade representation of a vertex by the blade representation of the digit being flipped.

Given a random variable $\xi$, the expectation of $\xi$ will be denoted by either $\langle\xi\rangle$ or $\mathbb{E}(\xi)$. Given a sequence of random variables $\left\{\xi_{N}\right\}$, the notation $\xi_{N} \stackrel{\mathcal{D}}{\rightarrow} \psi$ denotes convergence in distribution to the random variable $\psi$. The notation $\xi_{N} \stackrel{\mathcal{P}}{\rightarrow} u$ denotes convergence in probability to $u$.

Fix nonnegative integers $p$ and $q$, and let $n=p+q$. Following the approach of Guillotin-Plantard and Schott [4], let $\Sigma=(E, \mathcal{A}, \mu, T)$ be a dynamical system where $(E, \mathcal{A}, \mu)$ is a probability space and $T$ is a transformation on $E$.

Let $x \in E$, and define the collection $\left\{p_{j}\right\}_{1 \leq j \leq N^{d}}$ such that $0 \leq p_{j}<\frac{1}{N^{d}}$ for each $j=1,2, \ldots, N^{d}$ and $\sum_{j=1}^{N^{d}} p_{j} \leq 1$. Further, define the collection $\left\{f_{j}(x)\right\}_{1 \leq j \leq N^{d}}$ such that for each $i \geq 1$

$$
0 \leq f_{j}\left(T^{i} x\right) \leq p_{j}
$$

and define

$$
f_{0}\left(T^{i} x\right)=1-\sum_{j=1}^{N^{d}} f_{j}\left(T^{i} x\right)
$$

For $n \geq 1$, let $\left\{Y_{1}, \ldots, Y_{n}\right\}$ be i.i.d. random variables taking values in $\left\{0, \ldots, N^{d}\right\}$ with probability

$$
\mathbb{P}\left(Y_{i}=\ell\right)=f_{\ell}\left(T^{i} x\right)
$$

We are interested in the dynamic random walk on $\mathcal{C} \ell_{V}{ }^{\text {sym }}$ defined by

$$
\Upsilon_{n}=\prod_{k=1}^{n} \varsigma_{\left\{Y_{k}\right\}}
$$


Note that when $\sum_{j=1}^{N^{d}} p_{j}=1$, the resulting walk $\left(\Upsilon_{n}\right)$ is represented by a walk on the $N^{d}$-dimensional hypercube $\mathcal{Q}_{N^{d}}$, while $\sum_{j=1}^{N^{d}} p_{j}<1$ results in a walk on a hypercube with loops, denoted $\tilde{\mathcal{Q}}_{N^{d}}$. Figure 1 illustrates these two cases.

Using the one-to-one correspondence between multivectors $\varsigma_{U}$ and geometric graphs $G_{U}$, the walk $\left(\Upsilon_{n}\right)$ induces a dynamic random walk $\left(\mathcal{G}_{n}\right)$ on geometric graphs. Independence of the collection $\left\{f_{j}\left(T^{i} x\right)\right\}$ then gives

$$
\mathbb{P}\left(\mathcal{G}_{n}=G_{U}\right)=\left\langle\prod_{i=1}^{n}\left(f_{0}\left(T^{i} x\right)+\sum_{j=1}^{N^{d}} f_{j}\left(T^{i} x\right)_{\{j\}}\right), \varsigma_{U}\right\rangle
$$

For convenience, define the notation

$$
\tau_{n}=\prod_{i=1}^{n}\left(f_{0}\left(T^{i} x\right)+\sum_{j=1}^{N^{d}} f_{j}\left(T^{i} x\right) \varsigma_{\{j\}}\right)
$$

so that

$$
\left\langle\tau_{n}, \varsigma_{U}\right\rangle=\mathbb{P}\left(\mathcal{G}_{n}=G_{U}\right) .
$$

Theorem 3.1. Let $k \geq 3$ be fixed. Let $\Phi_{r}$ denote the second quantization nilpotent adjacency operator. Let $X_{k}(n)$ denote the number of $k$-cycles in the $n^{\text {th }}$ geometric graph of the sequence in the partitioned $d$-cube with mesh $1 / N^{d}$. Then,

$$
\mathbb{E}\left(X_{k}(n)\right)=\frac{1}{2 k} \sum_{U \in 2^{V}}\left\langle\tau_{n}, \varsigma_{U}\right\rangle \psi\left(\operatorname{Tr}_{U}\left(\Phi_{r}{ }^{k}\right)\right)
$$

Proof. For fixed $U \subseteq V$, a straightforward proof by induction shows that $\psi\left(\operatorname{Tr}_{U}\left(\Phi_{r}{ }^{k}\right)\right)$ is a sum of products of $k$ generators of $\mathcal{C} \ell_{V}{ }^{\text {nil }}$ corresponding to closed $k$-walks contained in $G_{U}$. Because $\zeta_{\{v\}}{ }^{2}=0$ for all $v \in V$, the only nonzero terms correspond to the $k$-cycles of $G_{U}$. Since each cycle has $k$ possible choices of basepoint and two orientations, it is evident that

$$
\psi\left(\operatorname{Tr}_{U}\left(\Phi_{r}{ }^{k}\right)\right)=2 k X_{k}(U)
$$

where $X_{k}(U)$ denotes the number of $k$-cycles contained in $G_{U}$. 
Now,

$$
\begin{array}{rl}
\frac{1}{2 k} \sum_{U \in 2^{V}}\left\langle\tau_{n}, \varsigma_{U}\right\rangle \psi & \left(\operatorname{Tr}_{U}\left(\Phi_{r}{ }^{k}\right)\right) \\
=\frac{1}{2 k} \sum_{U \in 2^{V}} & \mathbb{P}\left(\mathcal{G}_{n}=G_{U}\right) \psi\left(\operatorname{Tr}_{U}\left(\Phi_{r}{ }^{k}\right)\right) \\
\quad & =\sum_{U \in 2^{V}} \mathbb{P}\left(\mathcal{G}_{n}=G_{U}\right) X_{k}(U)=\mathbb{E}\left(X_{k}(n)\right)
\end{array}
$$

Example 3.2. To compute the expected number of 5-cycles in the $7^{\text {th }}$ graph of a dynamic geometric process in the partitioned unit square with mesh $1 / 9$, we begin by defining the probability functions $\left\{f_{j}(x)\right\}$. The ergodic mapping $T$ acts on $S^{1}$ according to $T x=e^{i} x$. Additional Mathematica procedures for computing products in $\mathcal{C} \ell_{n}{ }^{\text {nil }}$ and $\mathcal{C} \ell_{n}{ }^{\text {sym }}$ can be found in the appendix.

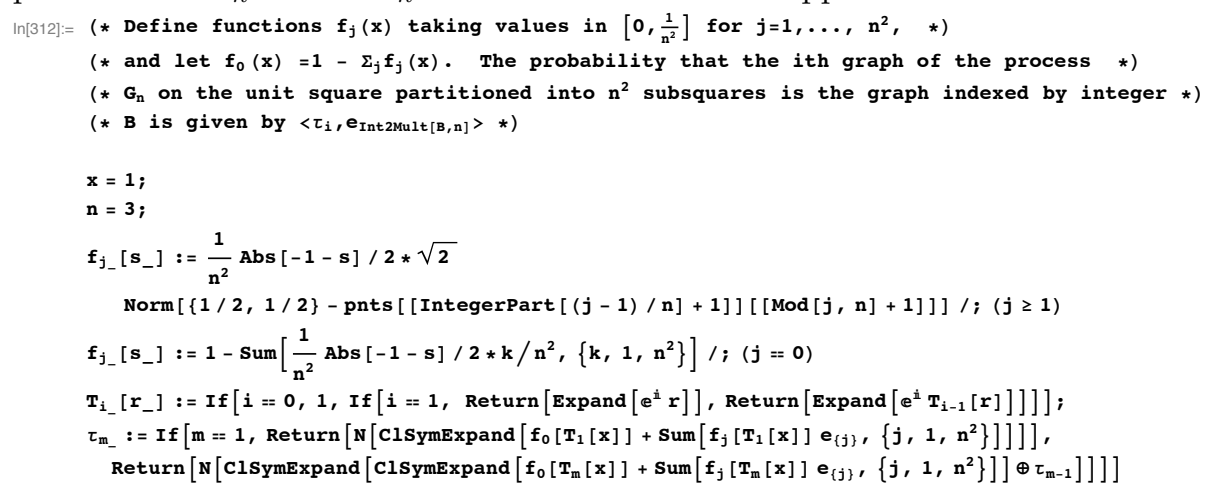

For simplicity, subsets of vertices are associated with binary representations of integers.

$\ln [4]:=$ ( * Convert binary string to coordinates of subsquare centers

in $[0,1]^{2}$ with mesh $1 / \mathrm{n}^{2}$. $\mathrm{B}$ is an integer in the range 0 to $2^{\mathrm{n}^{2}}-1 *$ )

Bin2Coord [B_, $\left.\mathrm{n}_{-}\right]:=$(

$\mathrm{d}=$ PadLeft $\left[\right.$ IntegerDigits $\left.[\mathrm{B}, 2], \mathrm{n}^{2}\right]$;

pnts $=$ Table $\left[\left\{\frac{(2 j-1)}{2 n}, \frac{(2 k-1)}{2 n}\right\},\{j, 1, n\},\{k, 1, n\}\right] ;$

$\mathbf{Y}=\operatorname{Table}\left[\mathbf{d}[[j]] \operatorname{pnts}[[\operatorname{IntegerPart}[(j-1) / \mathrm{n}]+1]][[\operatorname{Mod}[j, \mathbf{n}]+1]],\left\{j, 1, \mathbf{n}^{2}\right\}\right]$;

Return [DeleteCases $[Y,\{0,0\}]]$;

)

The following Mathematica procedure generates the nilpotent adjacency matrix associated with a subset of vertices. 


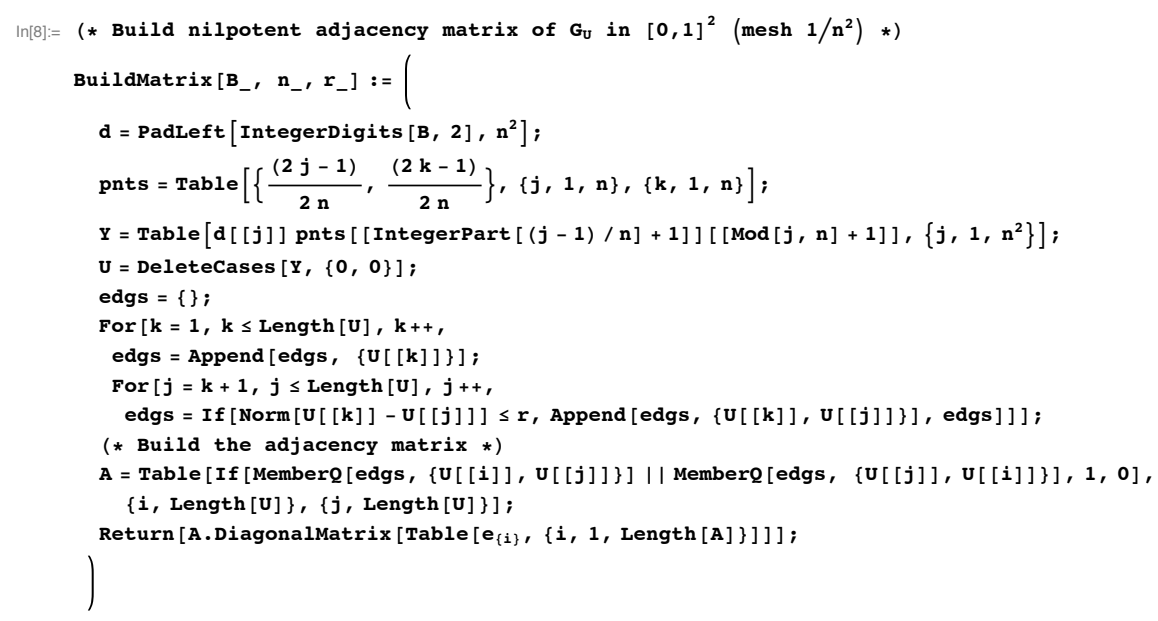

We now generate nilpotent adjacency matrices for all subsets.

$\ln [1]\}=\left(*\right.$ Build all possible adjacency matrices in $[0,1]^{2}$ with mesh $1 / 9$ and $\left.r=\sqrt{2} / 3 *\right)$

$\Lambda_{0}=\{\{0\}\}$;

For $[\mathrm{m}=1, \mathrm{~m} \leq 511, \mathrm{~m}++$,

$\Lambda_{\mathrm{m}}=$ BuildMatrix $[\mathrm{m}, 3, \sqrt{2} / 3]$;

]

The next procedure converts an integer into its appropriate multiindex.

$\operatorname{In}[226]:=\operatorname{Int2Mult}\left[B_{-}, n_{-}\right]:=\operatorname{DeleteCases}\left[\operatorname{Table}\left[\operatorname{If}\left[\operatorname{BitAnd}\left[B_{,} 2^{j}\right]=2^{j}, j+1,0\right],\left\{j, 0, n^{2}-1\right\}\right], 0\right]$

Finally, we compute the expected number of 5 -cycles in $\mathcal{G}_{7}$.

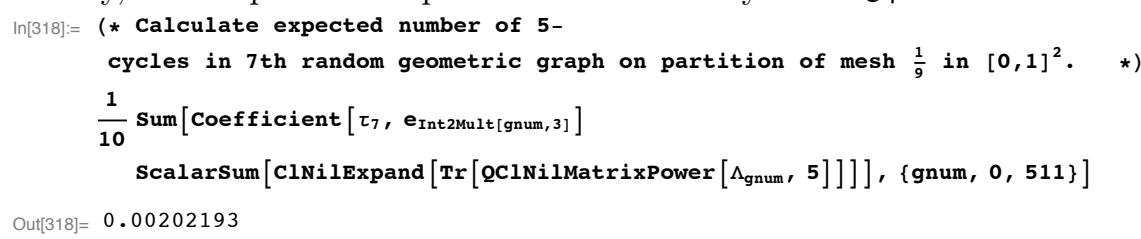

Corollary 3.3. The probability that the $n^{\text {th }}$ random geometric graph of a sequence contains exactly $\ell$ cycles of length $k$ is given by

$$
\mathbb{P}\left(X_{k}(n)=\ell\right)=\sum_{U \in 2^{V}}\left\langle e_{\psi\left(\operatorname{Tr}_{U}\left(\Phi_{r}{ }^{k}\right)\right)}, e_{2 k \ell}\right\rangle\left\langle\tau_{n}, \varsigma_{U}\right\rangle
$$

Proof. Note that the geometric graph $G_{U}$ contains $\ell k$-cycles if and only if $\psi\left(\operatorname{Tr}_{U}\left(\Phi_{r}{ }^{k}\right)\right)=$ $2 k \ell$. Hence, $G_{U}$ contains $\ell k$-cycles if and only if

$$
\left\langle e_{\psi\left(\operatorname{Tr}_{U}\left(\Phi_{r}{ }^{k}\right)\right)}, e_{2 k \ell}\right\rangle=1
$$


Summing the probabilities $\mathbb{P}\left(\mathcal{G}_{n}=G_{U}\right)$ over all graphs $G_{U}$ gives the stated result.

Example 3.4. The probabilities of $\ell 5$-cycles in the seventh graph of the sequence considered in Example 3.2 are displayed for $0 \leq \ell \leq 10$.

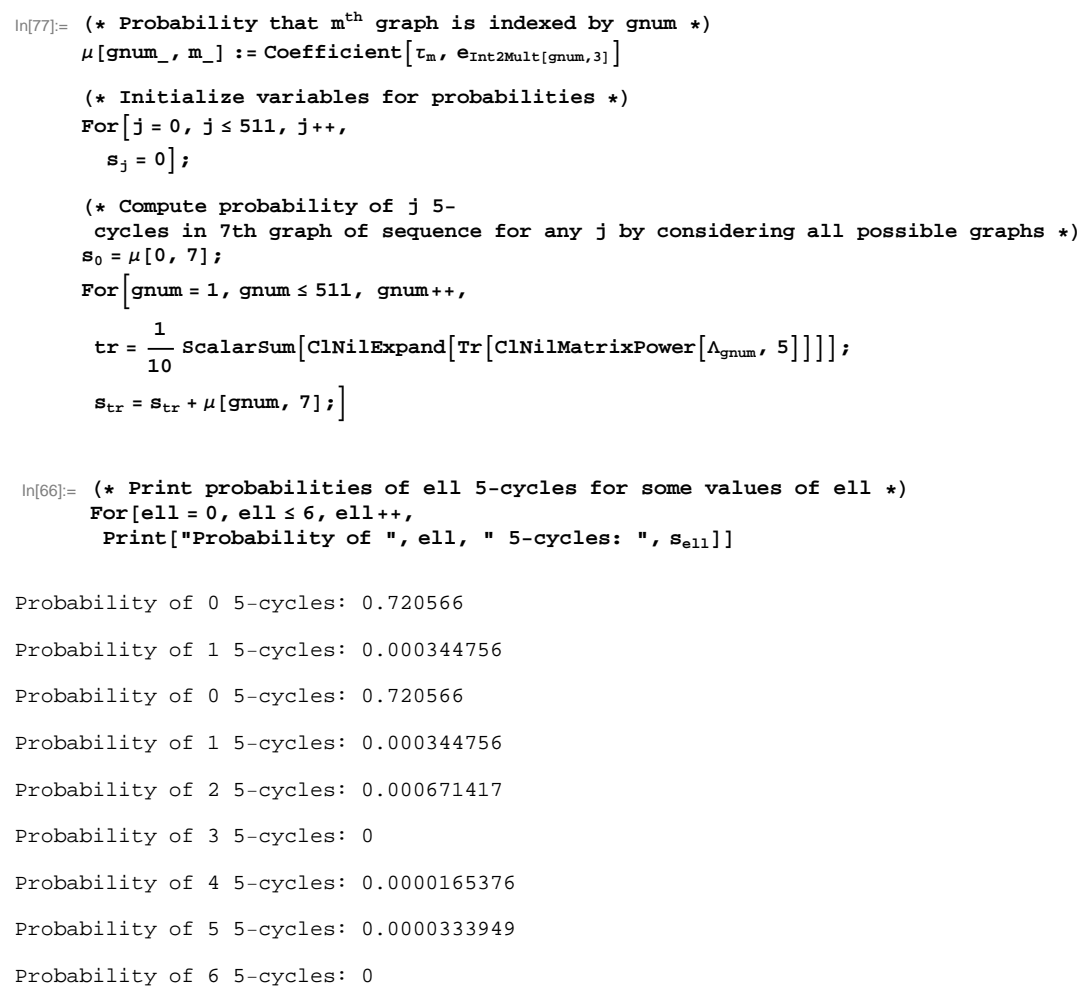

Recall that the circumference of a graph $G$ is the length of the longest cycle contained in $G$. Circumference will be denoted by $\operatorname{Circ}(G)$.

Lemma 3.5. Fix integer $\ell$ such that $3 \leq \ell \leq|V|$. Then, the geometric graph $G_{U}$ has circumference $\ell$ if and only if

$$
\left\langle e_{\psi\left(\operatorname{Tr}_{U}\left(\Phi_{r}{ }^{\ell}\right)\right)}, \vec{e}_{V}\right\rangle \prod_{k=\ell+1}^{|V|}\left\langle e_{\psi\left(\operatorname{Tr}_{U}\left(\Phi_{r}{ }^{k}\right)\right)}, e_{0}\right\rangle=1 .
$$

Proof. Note that $\left\langle e_{\psi\left(\operatorname{Tr}_{U}\left(\Phi_{r}{ }^{\ell}\right)\right)}, \vec{e}_{V}\right\rangle=1$ if and only if $G_{U}$ contains an $\ell$ cycle, while $\prod_{k=\ell+1}^{|V|}\left\langle e_{\psi\left(\operatorname{Tr}_{U}\left(\Phi_{r}{ }^{k}\right)\right)}, e_{0}\right\rangle=1$ if and only if $G_{U}$ does not contain a $k$ cycle for any $\ell<k \leq|V|$. 
The preceding lemma can be applied to compute the expected circumference of the $n^{\text {th }}$ graph of a process.

Proposition 3.6. The expected circumference of the $n^{\text {th }}$ geometric graph of the graph process is given by

$\mathbb{E}\left(\operatorname{Circ}\left(\mathcal{G}_{n}\right)\right)=\sum_{\ell=3}^{|V|} \ell\left(\sum_{U \in 2^{V}}\left\langle e_{\psi\left(\operatorname{Tr}_{U}\left(\Phi_{r}{ }^{\ell}\right)\right)}, \vec{e}_{V}\right\rangle \prod_{k=\ell+1}^{|V|}\left\langle e_{\psi\left(\operatorname{Tr}_{U}\left(\Phi_{r}{ }^{k}\right)\right)}, e_{0}\right\rangle\left\langle\tau_{n}, \varsigma_{U}\right\rangle\right)$.

Proof. In light of Lemma 3.5,

$$
\begin{array}{r}
\sum_{\ell=3}^{|V|} \ell\left(\sum_{U \in 2^{V}}\left\langle e_{\psi\left(\operatorname{Tr}_{U}\left(\Phi_{r}{ }^{\ell}\right)\right)}, \vec{e}_{V}\right\rangle \prod_{k=\ell+1}^{|V|}\left\langle e_{\psi\left(\operatorname{Tr}_{U}\left(\Phi_{r}{ }^{k}\right)\right)}, e_{0}\right\rangle\left\langle\tau_{n}, \varsigma_{U}\right\rangle\right) \\
=\sum_{\ell=3}^{|V|} \sum_{\left\{U: \operatorname{Circ}\left(G_{U}\right)=\ell\right\}} \mathbb{P}\left(\mathcal{G}_{n}=G_{U}\right) \\
=\sum_{\ell=3}^{|V|} \ell \mathbb{P}\left(\operatorname{Circ}\left(\mathcal{G}_{n}\right)=\ell\right) .
\end{array}
$$

A concept "dual" to graph circumference is graph girth. Recall that the girth of a graph $G$ is the length of the shortest cycle contained in $G$. Girth will be denoted by $\operatorname{Girth}(G)$.

Lemma 3.7. Fix integer $\ell$ such that $3 \leq \ell \leq|V|$. Then, the geometric graph $G_{U}$ has girth $\ell$ if and only if

$$
\left\langle e_{\psi\left(\operatorname{Tr}_{U}\left(\Phi_{r}{ }^{\ell}\right)\right)}, \vec{e}_{V}\right\rangle \prod_{k=3}^{\ell-1}\left\langle e_{\psi\left(\operatorname{Tr}_{U}\left(\Phi_{r}{ }^{k}\right)\right)}, e_{0}\right\rangle=1
$$

Proof. The proof is analogous to that of Lemma 3.5.

This necessary and sufficient condition can now be used to compute the expected girth of the $n^{\text {th }}$ graph of a graph process.

Proposition 3.8. The expected girth of the $n^{\text {th }}$ random geometric graph $\mathcal{G}_{n}$ is given by

$$
\mathbb{E}\left(\operatorname{Girth}\left(\mathcal{G}_{n}\right)\right)=\sum_{\ell=3}^{|V|} \ell\left(\sum_{U \in 2^{V}}\left\langle e_{\psi\left(\operatorname{Tr}_{U}\left(\Phi_{r}{ }^{\ell}\right)\right)}, \vec{e}_{V}\right\rangle \prod_{k=2}^{\ell-1}\left\langle e_{\psi\left(\operatorname{Tr}_{U}\left(\Phi_{r}{ }^{k}\right)\right)}, e_{0}\right\rangle\left\langle\tau_{n}, \varsigma_{U}\right\rangle\right) .
$$


Proof. In light of Lemma 3.7,

$$
\begin{aligned}
& \sum_{\ell=3}^{|V|} \ell\left(\sum_{U \in 2^{V}}\left\langle e_{\psi\left(\operatorname{Tr}_{U}\left(\Phi_{r}{ }^{\ell}\right)\right)}, \vec{e}_{V}\right\rangle \prod_{k=2}^{\ell-1}\left\langle e_{\psi\left(\operatorname{Tr}_{U}\left(\Phi_{r}{ }^{k}\right)\right)}, e_{0}\right\rangle\left\langle\tau_{n}, \varsigma_{U}\right\rangle\right) \\
&=\sum_{\ell=3}^{|V|} \ell \sum_{\left\{U: \operatorname{Girth}\left(G_{U}\right)=\ell\right\}} \mathbb{P}\left(\mathcal{G}_{n}=G_{U}\right) \\
& \\
&=\sum_{\ell=3}^{|V|} \ell \mathbb{P}\left(\operatorname{Girth}\left(\mathcal{G}_{n}\right)=\ell\right) .
\end{aligned}
$$

Of particular interest in models of wireless networks is the size of a graph's largest connected component. The size is expressed as the number of vertices contained in the component.

Proposition 3.9. The size of the largest component $C_{\max }$ in $G_{U}$ is given by

$$
\left|C_{\max }\right|=\delta\left(\operatorname{Tr}_{U}\left(\Xi_{r}^{2|U|-1}\right)\right) .
$$

Proof. An inductive argument shows that the terms of $\operatorname{Tr}_{U}\left(\Xi_{r}^{2 n-1}\right)$ are sums of idempotents representing closed walks of length $2 n-1$ on the graph. Because the graph contains $|U|$ vertices, the maximal connected component of $G$ will be covered by a closed walk of length $2|U|-1$ or less. All components can be covered by closed walks of length equal to $2|U|-1$ by the inclusion of a loop based at each vertex in the definition of the idempotent-adjacency operator.

Clearly, a connected graph consists of a single component containing all of the graph's vertices. The following corollary is an immediate consequence of Proposition 3.9 .

Corollary 3.10. The graph $G_{U}$ on vertices $U \in 2^{V}$ is connected if and only if

$$
\delta\left(\left\langle e_{U, j}\left|\Xi_{r}^{2|V|-1}\right| e_{U, j}\right\rangle\right)=|U|,
$$

for any $v_{j} \in U$.

The expected maximal component size in a graph of a process can also be computed.

Proposition 3.11. The expected size of a maximal component in the $n^{\text {th }}$ geometric graph of the process is given by

$$
\mathbb{E}\left(\left|C_{\max }\right|\right)=\sum_{U \in 2^{V}}\left\langle\tau_{n}, \varsigma_{U}\right\rangle \delta\left(\operatorname{Tr}_{U}\left(\Xi_{r}^{2|V|-1}\right)\right) .
$$


Proof. In light of Proposition 3.9,

$$
\sum_{U \in 2^{V}}\left\langle\tau_{n}, \varsigma_{U}\right\rangle \delta\left(\operatorname{Tr}_{U}\left(\Xi_{r}^{2|V|-1}\right)\right)=\sum_{\left\{U: G_{U} \text { is connected }\right\}}|U| \cdot \mathbb{P}\left(\mathcal{G}_{n}=G_{U}\right) .
$$

Another question of interest in the wireless network model context is whether or not a connected graph contains small cycles. Ideally, a network should be connected without too much redundancy (or too many unnecessary connections). The next result gives the probability that a graph of the process is connected and contains no cycles of length less than or equal to $k_{0}$.

Proposition 3.12. Let $\mathcal{G}_{n}$ be the $n^{\text {th }}$ graph of a geometric graph process, and let $k_{0} \geq 3$ be an integer. Then,

$\mathbb{P}\left(\mathcal{G}_{n}\right.$ connected and contains no $k$-cycles for all $\left.k \leq k_{0}\right)$

$$
=\sum_{U \in 2^{V}}\left\langle\tau_{n}, \varsigma_{U}\right\rangle\left\langle e_{\delta\left(\operatorname{Tr}_{U}\left(\Xi_{r}^{2|V|-1}\right)\right)}, e_{|U|}\right\rangle\left\langle e_{\sum_{k=3}^{k_{0}} \psi\left(\operatorname{Tr}_{U}\left(\Phi_{r}{ }^{k}\right)\right)}, e_{0}\right\rangle .
$$

Proof. Note that for fixed $U \subseteq V, G_{U}$ contains no $k$-cycles for all $3 \leq k \leq k_{0}$ if and only if

$$
\left\langle e_{\sum_{k=3}^{k_{0}} \psi\left(\operatorname{Tr}_{U}\left(\Phi_{r}{ }^{k}\right)\right)}, e_{0}\right\rangle=1
$$

Moreover, $G_{U}$ is connected if and only if

$$
\left\langle e_{\delta\left(\operatorname{Tr}_{U}\left(\Xi_{r}^{2|V|-1}\right)\right)}, e_{|U|}\right\rangle=1 .
$$

Hence,

$$
\begin{aligned}
\sum_{U: \in 2^{V}}\left\langle\tau_{n}, \varsigma_{U}\right\rangle\left\langle e_{\delta\left(\operatorname{Tr}_{U}\left(\Xi_{r}{ }^{2|V|-1}\right)\right)}, e_{|U|}\right\rangle\left\langle e_{\sum_{k=3}^{k_{0}} \psi\left(\operatorname{Tr}_{U}\left(\Phi_{r}{ }^{k}\right)\right)}, e_{0}\right\rangle \\
=\sum_{\substack{\left\{U: G_{U} \text { connected and } \\
\text { contains no } k \text {-cycles } \forall k \leq k_{0}\right\}}} \mathbb{P}\left(\mathcal{G}_{n}=G_{U}\right) .
\end{aligned}
$$

The following corollary deals with spanning trees, i.e. cycle-free connected graphs.

Corollary 3.13. The probability that the $n^{\text {th }}$ geometric graph of the process is a spanning tree is given by

$$
\sum_{U \in 2^{V}}\left\langle\tau_{n}, \varsigma_{U}\right\rangle\left\langle e_{\delta\left(\operatorname{Tr}_{U}\left(\Xi_{r}^{2|V|-1}\right)\right)}, e_{|U|}\right\rangle\left\langle e_{\sum_{k=3}^{|U|} \psi\left(\operatorname{Tr}_{U}\left(\Phi_{r}{ }^{k}\right)\right)}, e_{0}\right\rangle .
$$


One final goal is to enumerate the connected components in a geometric graph $G_{U}$. To this end, let $x \in \mathcal{C} \ell_{V}{ }^{\text {idem }}$ and define the mapping $\eta: \mathcal{C} \ell_{V}{ }^{\text {idem }} \rightarrow \mathcal{C} \ell_{V}{ }^{\text {idem }}$ by

$$
\eta(x)=\eta\left(\sum_{U \in V} \alpha_{U} \gamma_{U}\right)=\sum_{|U|=\delta(x)} \alpha_{U} \gamma_{U}
$$

Define the function $\rho: \mathcal{C} \ell_{V}{ }^{\text {idem }} \rightarrow \mathbb{N}_{0}$ by

$$
\rho(x)=\min _{W \ni x}\{\operatorname{dim}(W)\} .
$$

In other words, $\rho(x)$ is the dimension of the smallest linear subspace of $\mathcal{C} \ell_{V}{ }^{\text {idem }}$ containing $x$. Now in a manner similar to the enumeration of cycles, it is possible to enumerate components.

Proposition 3.14. Let $\Xi_{r}$ denote the second quantization nilpotent adjacency operator. Let $X$ denote the number of connected components in the $n^{\text {th }}$ graph in the partitioned $d$-cube with mesh $1 / N^{d}$. Then,

$$
\mathbb{E}(X)=\sum_{U \in 2^{V}}\left\langle\tau_{n}, \varsigma_{U}\right\rangle \rho\left(\sum_{v_{j} \in U} \eta\left(\left\langle e_{U, j}\left|\Xi_{r}^{2|V|-1}\right| e_{U, j}\right\rangle\right)\right)
$$

Proof. Note that as in the proof of Proposition 3.9, the quantity $\left\langle e_{U, j}\left|\Xi_{r}{ }^{2|V|-1}\right| e_{U, j}\right\rangle$ is a sum of products of idempotents representing connected components containing vertex $v_{j}$ in the graph $G_{U}$. Hence, $\eta\left(\left\langle e_{U, j}\left|\Xi_{r}{ }^{2|V|-1}\right| e_{U, j}\right\rangle\right)$ is a sum of terms representing maximal components containing vertex $v_{j}$ in graph $G_{U}$.

After summing over all vertices, the number of maximal components in the graph $G_{U}$ is given by the quantity $\rho\left(\sum_{v_{j} \in U} \eta\left(\left\langle e_{U, j}\left|\Xi_{r}^{2|V|-1}\right| e_{U, j}\right\rangle\right)\right)$ by construction of $\rho$.

As seen before, $\left\langle\tau_{n}, \varsigma_{U}\right\rangle=\mathbb{P}\left(\mathcal{G}_{n}=G_{U}\right)$. Summing over all graphs $G_{U}$ completes the proof.

In addition to computing the expected number of components, we can calculate the probability that a graph in the process contains exactly $\ell$ components for any nonnegative integer $\ell$.

Proposition 3.15. Let $X_{n}$ denote the number of components in the $n^{\text {th }}$ geometric graph of the process. The probability that $X_{n}=\ell$ is given by

$$
\mathbb{P}\left(X_{n}=\ell\right)=\sum_{U \in 2^{V}}\left\langle e_{\rho\left(\sum_{v_{j} \in U} \eta\left(\left\langle e_{U, j}\left|\Xi_{r}^{2|V|-1}\right| e_{U, j}\right\rangle\right)\right)}, e_{\ell}\right\rangle\left\langle\tau_{n}, \varsigma_{U}\right\rangle .
$$

Proof. By construction, $\left.\left\langle e_{\rho\left(\sum_{v_{j} \in U}\right.} \eta\left(\left\langle e_{U, j}\left|\Xi_{r}{ }^{2|V|-1}\right| e_{U, j}\right\rangle\right)\right), e_{\ell}\right\rangle=1$ if and only if $G_{U}$ contains $\ell$ components. Summing the probabilities of graphs $G_{U}$ containing $\ell$ components gives (3.38). 


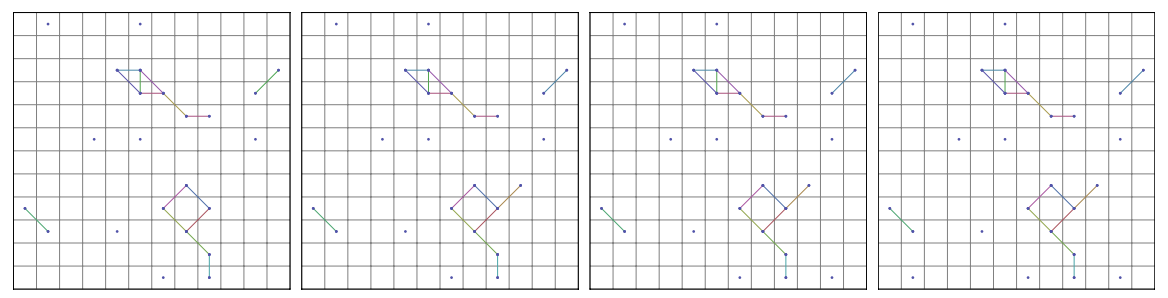

FiguRE 2. Four graphs of a dynamic geometric graph process.

The graphs on the partitioned cube can be partitioned into two sets: those that are connected, and those that are not.

Define $\vartheta \in \mathcal{C} \ell_{V}{ }^{\text {sym }}$ by

$$
\vartheta=\sum_{U \in 2^{V}}\left\langle e_{\delta\left(\operatorname{Tr}_{U}\left(\Xi_{r}^{2|V|-1}\right)\right)}, e_{|U|}\right\rangle \varsigma_{U} .
$$

Note that $\vartheta$ is a sum of blades indexed by vertex sets of connected graphs in the partitioned cube $[0,1]^{d}$. Hence,

$$
\left\langle\vartheta, \varsigma_{U}\right\rangle= \begin{cases}1 & \text { if } G_{U} \text { is connected } \\ 0 & \text { otherwise }\end{cases}
$$

Similarly, define

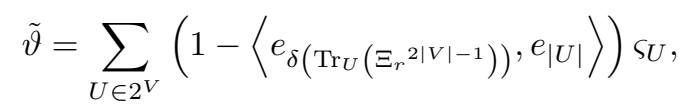

and note that $\tilde{\vartheta}$ is a sum of blades indexed by vertex sets of disconnected graphs in the partitioned cube $[0,1]^{d}$.

Often one wants to know the probability that a graph in the process is connected. The next result gives an expression for this probability.

Proposition 3.16. The probability that the $n^{\text {th }}$ graph of the process is connected is given by

$$
\mathbb{P}\left(\mathcal{G}_{n} \text { is connected }\right)=\left\langle\tau_{n}, \vartheta\right\rangle .
$$

Proof.

$$
\mathbb{P}\left(\mathcal{G}_{n} \text { is connected }\right)=\sum_{U \in 2^{V}}\left\langle\tau_{n}, \varsigma_{U}\right\rangle\left\langle\varsigma_{U}, \vartheta\right\rangle=\left\langle\tau_{n}, \vartheta\right\rangle
$$

Define the projection $\pi_{\tilde{\vartheta}}$ by

$$
\pi_{\tilde{\vartheta}}(x)=\sum_{\left\{U:\left\langle\varsigma_{U}, \vartheta\right\rangle=0\right\}} x_{U} \varsigma_{U} .
$$


Similarly, define $\pi_{\vartheta}$ by

$$
\pi_{\vartheta}(x)=\sum_{\left\{U:\left\langle\varsigma_{U}, \vartheta\right\rangle=1\right\}} x_{U} \varsigma_{U}
$$

For $n \geq 0$, define the mappings $\nu_{n}: \mathcal{C} \ell_{V}{ }^{\text {sym }} \rightarrow \mathcal{C} \ell_{V}{ }^{\text {sym }}$ by

$$
\begin{gathered}
\nu_{0}(x)=x \\
\nu_{1}(x)=\pi_{\vartheta}\left(x \tau_{1}\right) \\
\nu_{n}(x)=\pi_{\vartheta}\left(\nu_{n-1}(x) \tau_{n}\right)(n \geq 2) .
\end{gathered}
$$

Similarly, define the mappings $\tilde{\nu}_{n}: \mathcal{C} \ell_{V}{ }^{\text {sym }} \rightarrow \mathcal{C} \ell_{V}{ }^{\text {sym }}$ by

$$
\begin{gathered}
\tilde{\nu}_{0}(x)=x \\
\tilde{\nu}_{1}(x)=\pi_{\tilde{\vartheta}}\left(x \tau_{1}\right), \\
\tilde{\nu}_{n}(x)=\pi_{\tilde{\vartheta}}\left(\tilde{\nu}_{n-1}(x) \tau_{n}\right)(n \geq 2) .
\end{gathered}
$$

Proposition 3.17. Let $\mathcal{G}_{0}=G_{U}$ be a connected geometric graph, and let $n$ be a positive integer. The probability that $\mathcal{G}_{k}$ is connected for all $0 \leq k \leq n$ is

$$
\mathbb{P}\left(\mathcal{G}_{0}, \ldots, \mathcal{G}_{n} \text { connected }\right)=\left\langle\nu_{n}\left(\varsigma_{U}\right), \vartheta\right\rangle .
$$

Similarly, when $\mathcal{G}_{0}=G_{U}$ is a disconnected geometric graph, the probability that $\mathcal{G}_{k}$ is disconnected for all $0 \leq k \leq n$ is

$$
\mathbb{P}\left(\mathcal{G}_{0}, \ldots, \mathcal{G}_{n} \text { disconnected }\right)=\left\langle\tilde{\nu}_{n}\left(\varsigma_{U}\right), \tilde{\vartheta}\right\rangle .
$$

Proof. Let $\mathcal{G}_{0}=G_{U}$ be a connected geometric graph. Proof of the first part of the proposition is by induction on $n \geq 1$. When $n=1$, definition of $\nu_{1}$ gives

$$
\begin{aligned}
\nu_{1}\left(\varsigma_{U}\right)=\pi_{\vartheta}\left(\varsigma_{U} \tau_{1}\right)=\pi_{\vartheta}\left(\sum_{v \in V \cup\{0\}} \mathbb{P}\left(\mathcal{G}_{1}=G_{U \triangle\{j\}}\right) \varsigma_{U \triangle\{v\}}\right) \\
=\sum_{\left\{U: G_{U} \text { connected }\right\}} \mathbb{P}\left(\mathcal{G}_{1}=G_{U}\right) \varsigma_{U} .
\end{aligned}
$$

Hence, $\left\langle\nu_{1}\left(\varsigma_{U}\right), \vartheta\right\rangle=\mathbb{P}\left(\mathcal{G}_{0}, \mathcal{G}_{1}\right.$ connected $)$. Now assume true for some positive integer $n$ and proceed by induction. For convenience, denote by $U_{n}$ the vertex set of $\mathcal{G}_{n}$; i.e., $\mathcal{G}_{n}=G_{U_{n}}$.

$$
\begin{aligned}
\nu_{n+1}\left(\varsigma_{U}\right) & =\pi_{\vartheta}\left(\nu_{n}\left(\varsigma_{U}\right) \tau_{n+1}\right) \\
=\pi_{\vartheta} & \left(\sum_{v \in V \cup\{0\}} \mathbb{P}\left(\mathcal{G}_{0}, \ldots, \mathcal{G}_{n} \text { connected and } \mathcal{G}_{n+1}=G_{U_{n} \triangle\{v\}}\right) \varsigma_{U_{n} \triangle\{v\}}\right) \\
\quad & \sum_{\left\{U: G_{U} \text { connected }\right\}} \mathbb{P}\left(\mathcal{G}_{0}, \ldots, \mathcal{G}_{n} \text { connected and } \mathcal{G}_{n+1}=G_{U}\right) \varsigma_{U} .
\end{aligned}
$$


Hence,

$$
\begin{array}{r}
\left\langle\nu_{n+1}\left(\varsigma_{U}\right), \vartheta\right\rangle=\sum_{\left\{U: G_{U} \text { connected }\right\}} \mathbb{P}\left(\mathcal{G}_{0}, \ldots, \mathcal{G}_{n} \text { connected and } \mathcal{G}_{n+1}=G_{U}\right) \\
=\mathbb{P}\left(\mathcal{G}_{0}, \ldots, \mathcal{G}_{n+1} \text { connected }\right) .
\end{array}
$$

The proof of the second part of the proposition follows from the same logic.

Given an initial graph that is not connected, the next two results deal with the emergence of a connected graph in the process.

Corollary 3.18. Let $\mathcal{G}_{0}=G_{U}$ be a disconnected geometric graph. For $n \geq 1$, the probability that $\mathcal{G}_{n}$ is the first connected graph of the process is given by

$$
\mathbb{P}\left(\mathcal{G}_{n} \text { first connected graph }\right)=\left\langle\tilde{\nu}_{n-1}\left(\varsigma_{U}\right) \tau_{n}, \vartheta\right\rangle .
$$

Corollary 3.19. Let $\mathcal{G}_{0}=G_{U}$ be a disconnected geometric graph. The expected time $n$ when the geometric graph $\mathcal{G}_{n}$ is the first connected graph of the process is given by

$$
\mathbb{E}\left(n: \mathcal{G}_{n} \text { first connected graph }\right)=\sum_{n \geq 1} n\left\langle\tilde{\nu}_{n-1}\left(\varsigma_{U}\right) \tau_{n}, \vartheta\right\rangle .
$$

Similarly, given an initial graph that is connected, the following results deal with the occurrence of the first disconnected graph in the process.

Corollary 3.20. Let $\mathcal{G}_{0}=G_{U}$ be a connected geometric graph. For $n \geq 1$, the probability that $\mathcal{G}_{n}$ is the first disconnected graph of the process is given by

$$
\mathbb{P}\left(\mathcal{G}_{n} \text { first disconnected graph }\right)=\left\langle\nu_{n-1}\left(\varsigma_{U}\right) \tau_{n}, \tilde{\vartheta}\right\rangle .
$$

Corollary 3.21. Let $\mathcal{G}_{0}=G_{U}$ be a connected geometric graph. The expected time $n$ when the geometric graph $\mathcal{G}_{n}$ is the first disconnected graph of the process is given by

$$
\mathbb{E}\left(n: \mathcal{G}_{n} \text { first disconnected graph }\right)=\sum_{n \geq 1} n\left\langle\nu_{n-1}\left(\varsigma_{U}\right) \tau_{n}, \tilde{\vartheta}\right\rangle .
$$

\subsection{Vertex degrees in $\mathcal{G}_{n}$}

Given a graph $G=(V, E)$, the degree of a vertex $v \in V$ is defined by

$$
\operatorname{deg}(v)=\mid \text { edges incident with } v \text { in } G\} \mid .
$$

The degree of a graph $G$ is defined by

$$
\operatorname{deg}(G)=\inf _{v \in G} \operatorname{deg}(v) .
$$

In the context of wireless networks, the vertex degree represents the number of nodes within a node's transmission range. The degree of a graph then represents the minimum number of nodes within transmission range of any node in the network.

The lemmas and propositions below follow immediately from definitions and results acquired thus far. 
Lemma 3.22. Let the geometric graph $G_{U}$ be given. By construction of the second quantization nilpotent adjacency operator $\Phi_{r}$, the degree of vertex $v_{j} \in U$ s given by

$$
\operatorname{deg}\left(v_{j}\right)=\psi\left(\left\langle e_{U, j}\left|\Phi_{r}{ }^{2}\right| e_{U, j}\right\rangle\right)
$$

It follows naturally that the degree of $G_{U}$ is given by

$$
\operatorname{deg}\left(G_{U}\right)=\inf _{j \in U} \psi\left(\left\langle e_{U, j}\left|\Phi_{r}^{2}\right| e_{U, j}\right\rangle\right)
$$

The preceding lemma can now be used to compute the probability that a graph in the process has degree $k$ for some nonnegative integer $k$.

Proposition 3.23. Let $\left(\mathcal{G}_{n}\right)$ be a geometric graph process associated with $\Phi_{r}$. Then, for nonnegative integer $k$, the probability that $\operatorname{deg}\left(\mathcal{G}_{n}\right)=k$ is given by

$$
\mathbb{P}\left(\operatorname{deg}\left(\mathcal{G}_{n}\right)=k\right)=\sum_{U \subseteq V}\left\langle\tau_{n}, \varsigma_{U}\right\rangle\left\langle e_{\inf _{j \in U} \psi\left(\left\langle e_{U, j}\left|\Phi_{r}{ }^{2}\right| e_{U, j}\right\rangle\right)}, e_{k}\right\rangle .
$$

In the next pair of propositions, the expected degree of a graph in a process and expected degree of a vertex in the $n^{\text {th }}$ graph of a process are calculated.

Proposition 3.24. Let $\left(\mathcal{G}_{n}\right)$ be a geometric graph process associated with $\Phi_{r}$. Then, the expected value of $\operatorname{deg}\left(\mathcal{G}_{n}\right)$ is given by

$$
\mathbb{E}\left(\operatorname{deg}\left(\mathcal{G}_{n}\right)\right)=\sum_{U \subseteq V}\left\langle\tau_{n}, \varsigma_{U}\right\rangle \inf _{j \in U} \psi\left(\left\langle e_{U, j}\left|\Phi_{r}{ }^{2}\right| e_{U, j}\right\rangle\right)
$$

Proposition 3.25. Let $\left(\mathcal{G}_{n}\right)$ be a geometric graph process associated with $\Phi_{r}$. Then randomly choosing a vertex $v$ from the vertices of $\mathcal{G}_{n}$ with all vertices equiprobable,

$$
\mathbb{E}(\operatorname{deg}(v))=\sum_{U \subseteq V}\left\langle\tau_{n}, \varsigma_{U}\right\rangle \psi\left(\operatorname{Tr}\left(\Phi_{r}^{2}\right)\right)
$$

A number of results involving conditional probabilities and conditional expectations are now possible.

Proposition 3.26. Let $\left(\mathcal{G}_{n}\right)$ be a geometric graph process associated with $\Phi_{r}$, let $k$ be a nonnegative integer, and let $\vartheta$ be defined as in (3.39). Then the conditional probability that $\mathcal{G}_{n}$ has minimum vertex degree $k$ given that $\mathcal{G}_{n}$ is connected is given by

$$
\mathbb{P}\left(\operatorname{deg}\left(\mathcal{G}_{n}\right)=k \mid \mathcal{G}_{n} \text { is connected }\right)=\frac{\sum_{U \subseteq V}\left\langle\vartheta, \varsigma_{U}\right\rangle\left\langle\tau_{n}, \varsigma_{U}\right\rangle\left\langle e_{\operatorname{deg}\left(G_{U}\right)}, e_{k}\right\rangle}{\sum_{U \subseteq V}\left\langle\vartheta, \varsigma_{U}\right\rangle\left\langle\tau_{n}, \varsigma_{U}\right\rangle} .
$$

Corollary 3.27. Let $\left(\mathcal{G}_{n}\right)$ be a geometric graph process associated with $\Phi_{r}$, and let $\vartheta$ be defined as in (3.39). Then the conditional expected value of $\operatorname{deg}\left(\mathcal{G}_{n}\right)$ given that $\mathcal{G}_{n}$ is connected is given by

$$
\mathbb{E}\left(\operatorname{deg}\left(\mathcal{G}_{n}\right) \mid \mathcal{G}_{n} \text { is connected }\right)=\frac{\sum_{U \subseteq V} \operatorname{deg}\left(G_{U}\right)\left\langle\vartheta, \varsigma_{U}\right\rangle\left\langle\tau_{n}, \varsigma_{U}\right\rangle}{\sum_{U \subseteq V}\left\langle\vartheta, \varsigma_{U}\right\rangle\left\langle\tau_{n}, \varsigma_{U}\right\rangle} .
$$


Proposition 3.28. Let $\left(\mathcal{G}_{n}\right)$ be a geometric graph process associated with $\Phi_{r}$, let $k$ be a nonnegative integer, and let $\vartheta$ be defined as in (3.39). Then the conditional probability that $\mathcal{G}_{n}$ is connected given that it has minimum vertex degree $k$ is given by,

$$
\mathbb{P}\left(\mathcal{G}_{n} \text { is connected } \mid \operatorname{deg}\left(\mathcal{G}_{n}\right)=k\right)=\frac{\sum_{U \subseteq V}\left\langle\vartheta, \varsigma_{U}\right\rangle\left\langle\tau_{n}, \varsigma_{U}\right\rangle\left\langle e_{\operatorname{deg}\left(G_{U}\right)}, e_{k}\right\rangle}{\sum_{U \subseteq V}\left\langle e_{\operatorname{deg}\left(G_{U}\right)}, e_{k}\right\rangle\left\langle\tau_{n}, \varsigma_{U}\right\rangle} .
$$

Proposition 3.29. Let $\left(\mathcal{G}_{n}\right)$ be a geometric graph process associated with $\Xi_{r}$. Let $k$ be a nonnegative integer, and let $X_{n}$ denote the number of connected components in the $n^{\text {th }}$ graph of the process. Then,

$$
\begin{aligned}
& \mathbb{E}\left(X_{n} \mid \operatorname{deg}\left(\mathcal{G}_{n}\right)=k\right) \\
& =\frac{\sum_{U \in 2^{V}}\left\langle\tau_{n}, \varsigma_{U}\right\rangle \rho\left(\sum_{v_{j} \in U} \eta\left(\left\langle e_{U, j}\left|\Xi_{r}{ }^{2|V|-1}\right| e_{U, j}\right\rangle\right)\right)\left\langle e_{\operatorname{deg}\left(G_{u}\right)}, e_{k}\right\rangle}{\sum_{U \in 2^{V}}\left\langle\tau_{n}, \varsigma_{U}\right\rangle\left\langle e_{\operatorname{deg}\left(G_{u}\right)}, e_{k}\right\rangle} .
\end{aligned}
$$

\subsection{Energy and Laplacian Energy of Geometric Graphs}

Given the adjacency matrix $A$ associated with a graph $G$ on $n$ vertices, the energy of $G$ is defined as the sum of the singular values of $A$ :

$$
\mathcal{E}(G)=\sum_{i=1}^{n}\left|\lambda_{i}\right|
$$

where each $\lambda_{i}$ is an eigenvalue of $A$. It is evident that

$$
\operatorname{Tr}\left(A^{2}\right)=\sum_{i=1}^{|V|} \lambda_{i}^{2}=2|E|
$$

Let $\lambda_{1}, \ldots, \lambda_{|V|}$ denote the spectrum of $A$. Since $A$ is symmetric, $A$ has a spectral decomposition $A \mathcal{U}=\mathcal{U} \Lambda$ where $\mathcal{U}$ is an orthogonal matrix and $\Lambda$ is a diagonal matrix with the eigenvalues of $A$ along the main diagonal. It follows that $\Lambda=\mathcal{U}^{-1} A \mathcal{U}$ so that the energy of $G$ is given by

$$
\mathcal{E}(G)=\operatorname{Tr}\left(\left(\left(\mathcal{U}^{-1} A \mathcal{U}\right)^{2}\right)^{1 / 2}\right) .
$$

For each subset of vertices $U \subseteq V$, denote the ordinary adjacency operator of the corresponding subgraph $G_{U}$ by $A^{(U)}$. In particular,

$$
A^{(U)}=\sum_{\substack{v_{i}, v_{j} \in U \\ 0<\left\|v_{1}-v_{2}\right\|<N r}}\left|e_{U, v_{1}}\right\rangle\left\langle e_{U, v_{2}}\right| .
$$

This adjacency operator is related to the corresponding nilpotent adjacency operator by

$$
A^{(U)} Z^{(U)}=\Phi_{r}^{(U)},
$$

where $Z^{(U)}$ is the diagonal matrix of generators of $\mathcal{C} \ell_{V}{ }^{\text {nil }}$ corresponding to the subset $U \subseteq V$. As before, each $A^{(U)}$ is symmetric and therefore has spectral decomposition of the form $A^{(U)} \mathcal{U}^{(U)}=\mathcal{U}^{(U)} \Lambda^{(U)}$. 
Given the second quantization nilpotent adjacency operator $\Phi_{r}$ associated with a graph process $\left(\mathcal{G}_{n}\right)$, define the ordinary second quantization adjacency operator $A_{r}$ associated with $\Phi_{r}$ by

$$
A_{r}=\sum_{U \in 2^{V}}\left(A^{(U)} \otimes\left|e_{U}\right\rangle\left\langle e_{U}\right|\right) .
$$

Similarly, the unitary second quantization operator $\mathcal{U}_{r}$ is defined by

$$
\mathcal{U}_{r}=\sum_{U \in 2^{V}}\left(\mathcal{U}^{(U)} \otimes\left|e_{U}\right\rangle\left\langle e_{U}\right|\right) .
$$

The following result is an immediate consequence of definitions.

Lemma 3.30. For subset $U \subset V$, the energy of the geometric graph $G_{U}$ is given by

$$
\mathcal{E}\left(G_{U}\right)=\operatorname{Tr}_{U}\left(\left(\left(\mathcal{U}_{r}^{-1} A_{r} \mathcal{U}_{r}\right)^{2}\right)^{1 / 2}\right) .
$$

As a corollary of previous results, we obtain the next result.

Proposition 3.31. Given geometric graph process $\left(\mathcal{G}_{n}\right)$ with associated ordinary second quantization adjacency operator $A_{r}$, the expected energy of the $n^{\text {th }}$ graph of the process is given by

$$
\mathbb{E}\left(\mathcal{E}\left(\mathcal{G}_{n}\right)\right)=\sum_{U \subseteq V}\left\langle\tau_{n}, \varsigma_{U}\right\rangle \operatorname{Tr}_{U}\left(\left(\left(\mathcal{U}_{r}^{-1} A_{r} \mathcal{U}_{r}\right)^{2}\right)^{1 / 2}\right) .
$$

Recall that by Lemma 3.22, the degree of vertex $v_{j} \in U \subseteq V$ is given by

$$
\operatorname{deg}\left(v_{j}\right)=\psi\left(\left\langle e_{U, j}\left|\Phi_{r}{ }^{2}\right| e_{U, j}\right\rangle\right) .
$$

Let $A^{(U)}$ denote the ordinary adjacency matrix of the graph $G_{U}$, and define the diagonal matrix of vertex degrees $D^{(U)}$ by

$$
\left(D^{(U)}\right)_{j j}=\psi\left(\left\langle e_{U, j}\left|\Phi_{r}{ }^{2}\right| e_{U, j}\right\rangle\right) .
$$

For each subset of vertices $U \subseteq V$, denote the combinatorial Laplacian of the corresponding subgraph $G_{U}$ by $L^{(U)}$. In particular,

$$
L^{(U)}=D^{(U)}-A^{(U)} .
$$

Given a graph $G$ on $n$ vertices and $m$ edges, the Laplacian spectrum of $G$ is the spectrum of its Laplacian matrix. The Laplacian spectrum consists of the numbers $\mu_{1} \geq \mu_{2} \geq \cdots \mu_{n} \geq 0$. The Laplacian energy of $G$ is defined by

$$
\mathcal{E}_{\mathcal{L}}(G)=\sum_{i=1}^{n}\left|\mu_{i}-\frac{2 m}{n}\right| .
$$

The Laplacian also has spectral decomposition of the form $L^{(U)} \mathcal{O}_{U}=\mathcal{O}_{U} \Lambda_{U}$ for orthogonal $\mathcal{O}_{U}$ and diagonal matrix $\Lambda_{U}$. 
Lemma 3.32. For subset $U \subset V$, the Laplacian energy of the geometric graph $G_{U}$ is given by

$$
\mathcal{L}_{\mathcal{E}}\left(G_{U}\right)=\operatorname{Tr}\left(\left(\left(\mathcal{O}_{U}^{-1} L^{(U)} \mathcal{O}_{U}-\frac{\operatorname{Tr}\left(L^{(U)}\right)}{|U|} I\right)^{2}\right)^{1 / 2}\right)
$$

Proof. By the handshaking theorem, the sum of vertex degrees is twice the number of edges.

Proposition 3.33. Given geometric graph process $\left(\mathcal{G}_{n}\right)$ with associated second quantization combinatorial Laplacian $L_{r}$, the expected Laplacian energy of the $n^{\text {th }}$ graph of the process is given by

$$
\mathbb{E}\left(\mathcal{E}_{\mathcal{L}}\left(\mathcal{G}_{n}\right)\right)=\sum_{U \subseteq V}\left\langle\tau_{n}, \varsigma_{U}\right\rangle \operatorname{Tr}\left(\left(\left(\mathcal{O}_{U}^{-1} L^{(U)} \mathcal{O}_{U}-\frac{\operatorname{Tr}\left(L^{(U)}\right)}{|U|} I\right)^{2}\right)^{1 / 2}\right)
$$

\section{Convergence Conditions and Limit Theorems}

In order to establish limit theorems for the results of the preceding section, it is necessary to establish convergence of the sequence $\left(\tau_{n}\right)$ in $\mathcal{C} \ell_{V}{ }^{\text {sym }}$.

The norm on $\mathcal{C} \ell_{V}{ }^{\text {sym }}$ defined by

$$
\left\|\sum_{U \subseteq V} \alpha_{U} \varsigma_{U}\right\|^{2}=\sum_{U \subseteq V} \alpha_{U}^{2}
$$

corresponds to the Euclidean norm when $\mathcal{C} \ell_{V}{ }^{\text {sym }}$ is viewed as a real $2^{|V|}$-dimensional vector space. A sequence $\left(\xi_{n}\right)$ in $\mathcal{C} \ell_{V}{ }^{\text {sym }}$ will be said to converge to $\xi \in \mathcal{C} \ell_{V}{ }^{\text {sym }}$ if for every $\epsilon>0, \exists k_{\epsilon} \in \mathbb{N}$ such that $\left\|\xi_{n}-\xi\right\|<\epsilon$ whenever $n \geq k_{\epsilon}$.

By definition of $\tau_{n}$,

$$
\tau_{n}=\prod_{i=1}^{n}\left(\sum_{j=0}^{N^{d}} f_{j}\left(T^{i} x\right) \varsigma_{\{j\}}\right)=\sum_{U \in 2^{V}} \mathbb{P}\left(\Upsilon_{n}=\varsigma_{U}\right) \varsigma_{U} .
$$

Theorem 4.1. Define the set

$$
J:=\left\{k \in V: f_{k}(x)>0 \forall x \in E\right\} .
$$

If $f_{0}(x)>0 \forall x \in E$, then

$$
\lim _{n \rightarrow \infty} \tau_{n}=\frac{1}{2^{|J|}}+\sum_{\left\{U \in 2^{V}: U \cap J \neq \emptyset\right\}} \frac{1}{2^{|J|}} \varsigma_{U} .
$$

Proof. Let $U \subseteq V$ and let $k \in V$. Note that if $k \notin U$ and $f_{k}(x)=0$ for all $x \in E$, then $\left\langle\tau_{n}, \varsigma_{U}\right\rangle=0$ for all $n$. 
Fix $i \in V$, and let $\left(\Upsilon_{n}\right)$ be the dynamic walk corresponding to the collection $\left\{f_{v}(x): v \in V\right\}$. Define the real-valued sequence $\left(x_{n}\right)$ by

$$
x_{n}:=\mathbb{P}\left(i \in L \mid \Upsilon_{n}=\varsigma_{L}\right), n \geq 0
$$

Then

$$
\begin{gathered}
x_{0}=0, \\
x_{1}=f_{i}(T x), \\
x_{n+1}=x_{n}\left(1-f_{i}\left(T^{n+1} x\right)\right)+\left(1-x_{n}\right) f_{i}\left(T^{n+1} x\right), \quad \forall n \geq 1 .
\end{gathered}
$$

It will be shown by induction that $x_{n}<1 / 2$ for all $n \geq 1$. By definition, $f_{i}(x)<\frac{1}{2}$ for all $x \in E$. Write $f_{i}\left(T^{n} x\right)=1 / 2-\kappa(n)$ where $\kappa(n)>0, \forall n$. In the basis step, $x_{1}=f_{i}(T x)<1 / 2$.

It is now assumed that $x_{n}<1 / 2$ for some $n \geq 1$. Then,

$$
\begin{aligned}
x_{n+1}=x_{n}\left(1-f_{i}\left(T^{n} x\right)\right)+ & \left(1-x_{n}\right) f_{i}\left(T^{n} x\right) \\
= & x_{n}\left(1-\frac{1}{2}+\kappa(n)\right)+\left(1-x_{n}\right)\left(\frac{1}{2}-\kappa(n)\right) \\
=\frac{x_{n}}{2}+x_{n} \kappa(n)+\frac{1}{2}-\frac{x_{n}}{2}-\kappa(n)+x_{n} \kappa(n) & \\
= & \frac{1}{2}-\kappa(n)+2 x_{n} \kappa(n) \\
& <\frac{1}{2}-\kappa(n)+\kappa(n)=\frac{1}{2} .
\end{aligned}
$$

Hence, $x_{n}<\frac{1}{2}$ for all $n \geq 1$. It will now be shown that $x_{n+1}>x_{n}$ for all $n \geq 1$. Using the recurrence (5.12), $x_{n}<\frac{1}{2}$ implies

$$
x_{n+1}=x_{n}+f_{i}\left(T^{n} x\right)-2 f_{i}\left(T^{n} x\right) x_{n}>x_{n}+f_{i}\left(T^{n} x\right)-f_{i}\left(T^{n} x\right)=x_{n} .
$$

Now the sequence $\left(x_{n}\right)$ converges to $\xi \in\left(0, \frac{1}{2}\right)$ by the Monotone Convergence Theorem. In particular, for any $n \in \mathbb{N}, \xi$ must satisfy

$$
\xi=\xi\left(1-f_{i}\left(T^{n} x\right)\right)+(1-\xi) f_{i}\left(T^{n} x\right)=\xi+f_{i}\left(T^{n} x\right)-2 \xi f_{i}\left(T^{n} x\right) .
$$

Hence, $\xi$ satisfies $0=f_{i}\left(T^{n} x\right)-2 \xi f_{i}\left(T^{n} x\right)=f_{i}\left(T^{n} x\right)(1-2 \xi)$, which implies $\xi=\frac{1}{2}$.

It is evident that $\xi$ represents the limit of the probability that vertex $i$ is present in the random geometric graph $\mathcal{G}_{n}$ as $n \rightarrow \infty$. Fixing $U \subseteq V$ and letting $J$ be the set described in the statement of the theorem, it is evident that the limiting distribution is uniform on the vertices of $J$; i.e.,

$$
\lim _{n \rightarrow \infty} \mathbb{P}\left(\Upsilon_{n}=\varsigma_{U}\right)=\left(\prod_{j \in J \cap U} \frac{1}{2}\right)\left(\prod_{j \in J \cap U^{\prime}}\left(1-\frac{1}{2}\right)\right)=\frac{1}{2^{|J|}} .
$$

The following theorem is a corollary of the previous section's results. 
Theorem 4.2. Assume the collection $\left\{f_{j}(x)\right\}_{j \in V}$ satisfies the hypotheses of Theorem 4.1 , and let $\left(\mathcal{G}_{n}\right)$ be the corresponding dynamic geometric graph process. Let $J$ be the set defined in the statement of Theorem 4.1. Let $\Phi_{r}$ denote the second quantization nilpotent adjacency operator, and let $\Xi_{r}$ denote the second quantization nilpotent adjacency operator associated with $\left(\mathcal{G}_{n}\right)$, respectively.

Let $k \geq 3$ be fixed, and let $z_{k}(n)$ denote the number of $k$-cycles in the $n^{\text {th }}$ geometric graph of the sequence in the partitioned $d$-cube with mesh $1 / N^{d}$. Let $\left|C_{\max }(n)\right|$ denote the size of a maximal component in the $n^{\text {th }}$ geometric graph of the process. Let $K(n)$ denote the number of connected components in the $n^{\text {th }}$ graph in the partitioned d-cube with mesh $1 / N^{d}$.

Then,

$$
\lim _{n \rightarrow \infty} \mathbb{E}\left(z_{k}(n)\right)=\frac{1}{k 2^{|J|+1}} \sum_{U \in 2^{V}} \psi\left(\operatorname{Tr}_{U}\left(\Phi_{r}{ }^{k}\right)\right),
$$

$\lim _{n \rightarrow \infty} \mathbb{E}\left(\operatorname{Cr}\left(\mathcal{G}_{n}\right)\right)=$

$$
\frac{1}{2^{|J|}} \sum_{\ell=3}^{|V|} \ell\left(\sum_{U \in 2^{V}}\left\langle e_{\psi\left(\operatorname{Tr}_{U}\left(\Phi_{r}{ }^{\ell}\right)\right)}, \vec{e}_{V}\right\rangle \prod_{k=\ell+1}^{|V|}\left\langle e_{\psi\left(\operatorname{Tr}_{U}\left(\Phi_{r}{ }^{k}\right)\right)}, e_{0}\right\rangle\right),
$$

$\lim _{n \rightarrow \infty} \mathbb{E}\left(\operatorname{Girth}\left(\mathcal{G}_{n}\right)\right)=$

$$
\begin{aligned}
& \frac{1}{2^{|J|}} \sum_{\ell=3}^{|V|} \ell\left(\sum_{U \in 2^{V}}\left\langle e_{\psi\left(\operatorname{Tr}_{U}\left(\Phi_{r} \ell\right)\right)}, \vec{e}_{V}\right\rangle \prod_{k=2}^{\ell-1}\left\langle e_{\psi\left(\operatorname{Tr}_{U}\left(\Phi_{r}{ }^{k}\right)\right)}, e_{0}\right\rangle\right), \\
& \lim _{n \rightarrow \infty} \mathbb{E}\left(\left|C_{\max }(n)\right|\right)=\frac{1}{2^{|J|}} \sum_{U \in 2^{V}} \delta\left(\operatorname{Tr}_{U}\left(\Xi_{r}^{2|V|-1}\right)\right),
\end{aligned}
$$

and

$$
\lim _{n \rightarrow \infty} \mathbb{E}(K(n))=\frac{1}{2^{|J|}} \sum_{U \in 2^{V}} \rho\left(\sum_{v_{j} \in U} \eta\left(\left\langle e_{U, j}\left|\Xi_{r}^{2|V|-1}\right| e_{U, j}\right\rangle\right)\right) .
$$

\section{Time-homogeneous walks on random geometric graphs}

In this section results are obtained for graph processes in which the vertex addition/deletion probabilities do not vary with time.

Proposition 5.1. Let $Y$ be a random variable taking values in $[|V|] \cup\{0\}$ with probabilities $p_{i}=\operatorname{Pr}\{Y=i\}$ for each $0 \leq i \leq|V|$, and let $\left\{Y_{k}\right\}_{k>0}$ be the sequence of independent random variables obtained from repeated observations of $Y$. Let $1=$ $\varsigma_{\emptyset} \in \mathcal{C} \ell_{V}{ }^{\text {sym }}$ represent the initial probability density on the vertices of $\mathcal{Q}_{|V|}$; i.e., the 
initial vertex corresponds to the empty graph. Let $\tau=\sum_{i=0}^{|V|} p_{i} \varsigma_{i}$. For convenience, $\varsigma_{\{0\}}$ is identified with $\varsigma_{\emptyset}$. Then for $k>0$, the probability density function $f_{k}$ on the power set $2^{V}$ at time $k$ is defined by

$$
f_{k}(U)=\left\langle\tau^{k}, \varsigma_{U}\right\rangle .
$$

Proof. For $k \geq 0$, let $\xi_{k} \in \mathcal{C} \ell_{V}{ }^{\text {sym }}$ be defined by

$$
\xi_{k}=\sum_{U \in 2^{V}} f_{k}(U) \varsigma_{U}
$$

and note that $\xi_{0}=\varsigma_{\emptyset}=1$. We show that $\xi_{k}=\tau^{k}$ for all $k \geq 1$.

We see that the probability density at time $k=1$ is given by

$$
\xi_{1}=\sum_{U \in 2^{V}} f_{1}(U) \varsigma_{U}=\sum_{U \in 2^{V}}\left\langle\tau, \varsigma_{U}\right\rangle \varsigma_{U}=\tau .
$$

Assuming true for $k$, using independence of $\left\{Y_{k}\right\}$, and proceeding by induction, we find

$$
\begin{aligned}
& \xi_{k+1}=\sum_{U \in 2^{V}} f_{k+1}(U) \varsigma_{U}=\sum_{U \in 2^{V}} \sum_{j=1}^{|V|} f_{k+1}(U \triangle\{j\}) \varsigma_{U \triangle\{j\}} \\
& =\sum_{U \in 2^{V}} f_{k}(U) \varsigma_{U} \sum_{j=1}^{|V|} p_{j} \varsigma_{\{j\}}=\xi_{k}\left(\sum_{j=1}^{|V|} p_{j} \varsigma_{\{j\}}\right)=\left(\sum_{j=1}^{|V|} p_{j} \varsigma_{\{j\}}\right)^{k}\left(\sum_{j=1}^{|V|} p_{j} \varsigma_{\{j\}}\right) \\
& =\tau^{k} \tau=\tau^{k+1} .
\end{aligned}
$$

When $p_{0}=0$, the resulting sequence $\left(\Upsilon_{n}\right)$ is a walk on the hypercube $\mathcal{Q}_{|V|}$. When $p_{0}>0,\left(\Upsilon_{n}\right)$ is a walk on a hypercube with loops.

In the homogeneous case, the sequence $\left(\tau_{n}\right)$ defined by (3.13) is replaced by $\left(\tau^{n}\right)$. All of the previous results hold as stated with this modification.

Example 5.2. Let $\left\{p_{0}, p_{1}, \ldots, p_{4}\right\}=\{.15, .1, .2, .3, .25\}$. The probability density on the collection of geometric graphs $G_{U}$ at time $t=32$ is determined by setting $\tau:=\sum_{i=0}^{4} p_{i} \varsigma_{\{i\}}$ and computing $\tau^{32}$ with Mathematica in Figure 3.

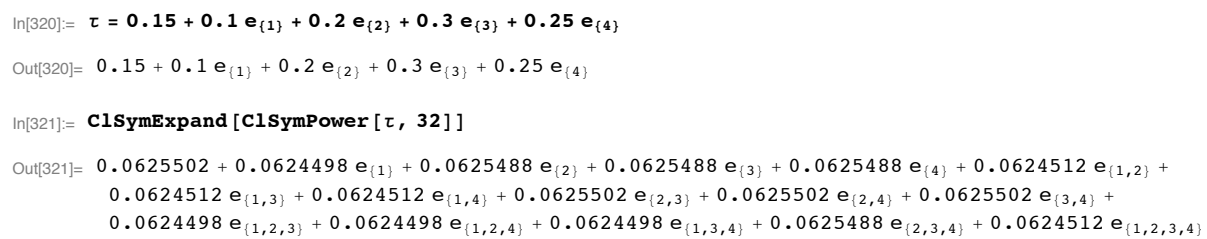

Figure 3. Probability that $\mathcal{G}_{32}=G_{U}$ for each $U \subseteq\left\{v_{1}, \ldots, v_{4}\right\}$. 
Now the probability that $\mathcal{G}_{32}=G_{\left\{v_{1}, v_{2}, v_{3}\right\}}$ is the coefficient of $\sigma_{\{1,2,3\}}$, seen to be .0624498 .

Lemma 5.3. Let $\left(\mathcal{G}_{n}\right)$ be the homogeneous geometric graph process defined in Proposition 5.1 with $p_{0}>0$, and let $i \in V$. Then, given $\mathcal{G}_{n}=G_{U_{n}}$,

$$
\mathbb{P}\left(i \in U_{n}\right)=\frac{1-\left(1-2 p_{i}\right)^{n}}{2}
$$

Proof. Fix $i \in V$, and let $\left(\Upsilon_{n}\right)$ be the time-homogeneous walk corresponding to the collection $\left\{p_{i}: v \in V\right\}$. The subset $U_{n} \subseteq V$ is defined by $\Upsilon_{n}=\varsigma_{U_{n}}$. Now define the sequence $\left(x_{n}\right)$ by $x_{n}=\mathbb{P}\left(i \in U_{n}\right)$ for $n \geq 0$. Then

$$
\begin{gathered}
x_{0}=0, \\
x_{n}=x_{n-1}\left(1-p_{i}\right)+\left(1-x_{n-1}\right) p_{i}, \quad \forall n \geq 1 .
\end{gathered}
$$

By back-substitution, the recurrence relation has solution given by

$$
\begin{gathered}
x_{n}=\left(1-2 p_{i}\right) x_{n-1}+p_{i} \\
=\left(1-2 p_{i}\right)^{2} x_{n-2}+\left(1-2 p_{i}\right) p_{i}+p_{i} \\
\vdots \\
=\left(1-2 p_{i}\right)^{n} x_{0}+p_{i} \sum_{j=0}^{n-1}\left(1-2 p_{i}\right)^{j} \\
=p_{i} \sum_{j=0}^{n-1}\left(1-2 p_{i}\right)^{j}=\frac{1-\left(1-2 p_{i}\right)^{n}}{2} .
\end{gathered}
$$

Theorem 5.4. Let $J=\left\{k \in V: p_{k}>0\right\}$. If $p_{0}>0$, then

$$
\lim _{n \rightarrow \infty} \tau^{n}=\frac{1}{2^{|J|}}+\sum_{\left\{U \in 2^{V}: U \cap J \neq \emptyset\right\}} \frac{1}{2^{|J|}} \varsigma_{U} .
$$

Proof. Let $U \subseteq V$ and let $k \in V$. Note that if $p_{k}=0$ and $k \notin U$, then $\left\langle\tau^{n}, \varsigma_{U}\right\rangle=0$ for all $n$.

Fix $i \in V$, and let $\left(\Upsilon_{n}\right)$ be the time-homogeneous walk corresponding to the collection $\left\{p_{i}: v \in V\right\} \cup\left\{p_{0}\right\}$. The subset $L \subseteq V$ is defined by $\Upsilon_{n}=\varsigma_{L}$.

Now define the sequence $\left(x_{n}\right)$ by $x_{n}=\mathbb{P}(i \in L)$ for $n \geq 0$. Then

$$
\begin{gathered}
x_{0}=0, \\
x_{1}=p_{i}, \\
x_{n+1}=x_{n}\left(1-p_{i}\right)+\left(1-x_{n}\right) p_{i}, \quad \forall n \geq 1 .
\end{gathered}
$$

Note that $p_{0}>0$ implies $p_{i}<1$. By Lemma 5.3,

$$
\lim _{n \rightarrow \infty} x_{n}=\lim _{n \rightarrow \infty} \frac{1-\left(1-2 p_{i}\right)^{n}}{2}=\frac{1}{2} \text {. }
$$


It is evident that $\frac{1}{2}$ represents the limit of the probability that vertex $i$ is present in the random geometric graph $\mathcal{G}_{n}$ as $n \rightarrow \infty$. Fixing $U \subseteq V$ and letting $J$ be the set described in the statement of the theorem, it is evident that the limiting distribution is uniform on the vertices of $J$; i.e.,

$$
\lim _{n \rightarrow \infty} \mathbb{P}\left(\Upsilon_{n}=\varsigma_{U}\right)=\left(\prod_{j \in J \cap U} \frac{1}{2}\right)\left(\prod_{j \in J \cap U^{\prime}}\left(1-\frac{1}{2}\right)\right)=\frac{1}{2^{|J|}} .
$$

\section{Mathematica Procedures}

This section contains general purpose Mathematica code for performing computations in the algebras $\mathcal{C} \ell_{n}{ }^{\text {nil }}$ and $\mathcal{C} \ell_{n}{ }^{\text {sym }}$. This code underlies the examples computed in the paper.

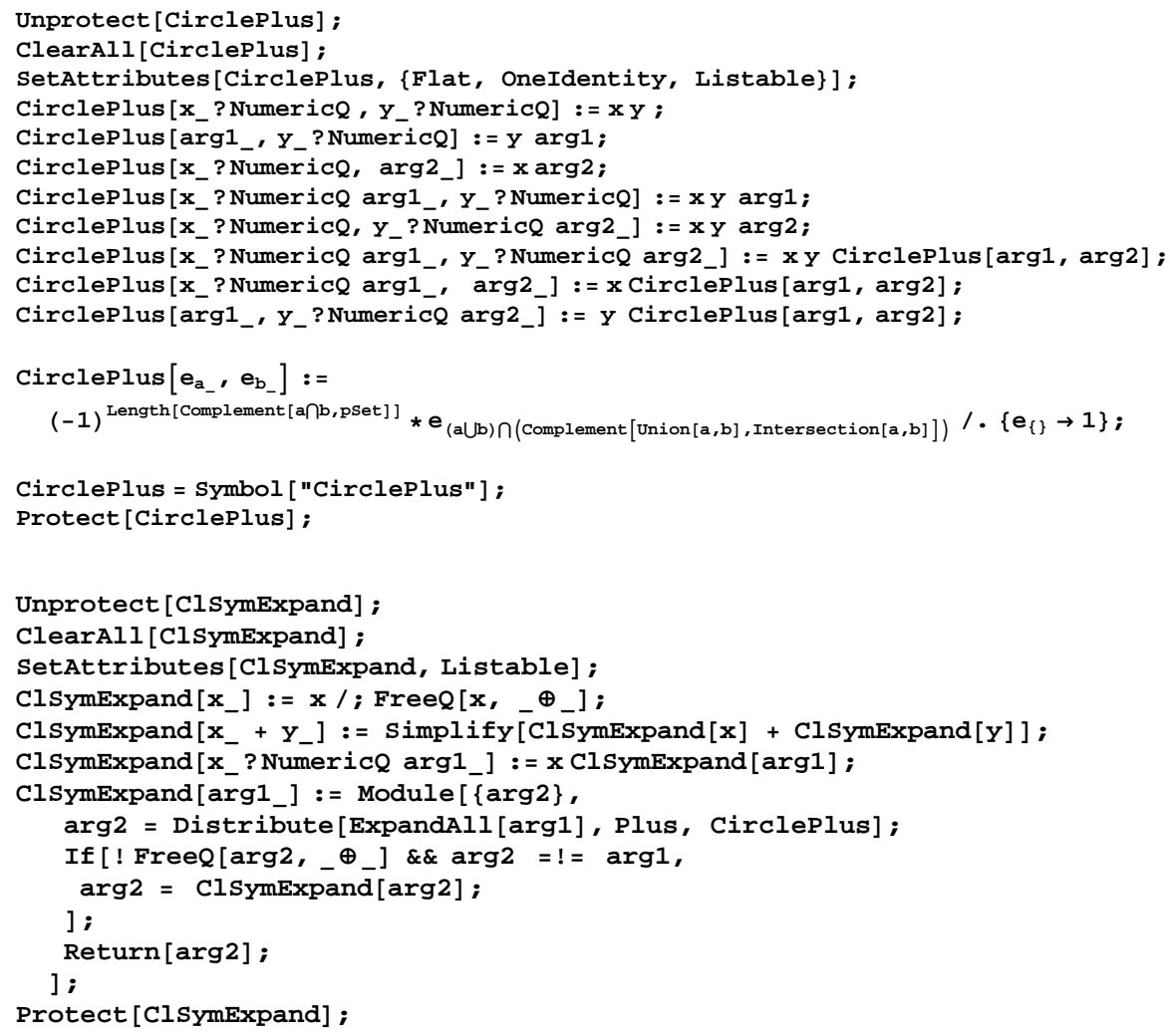




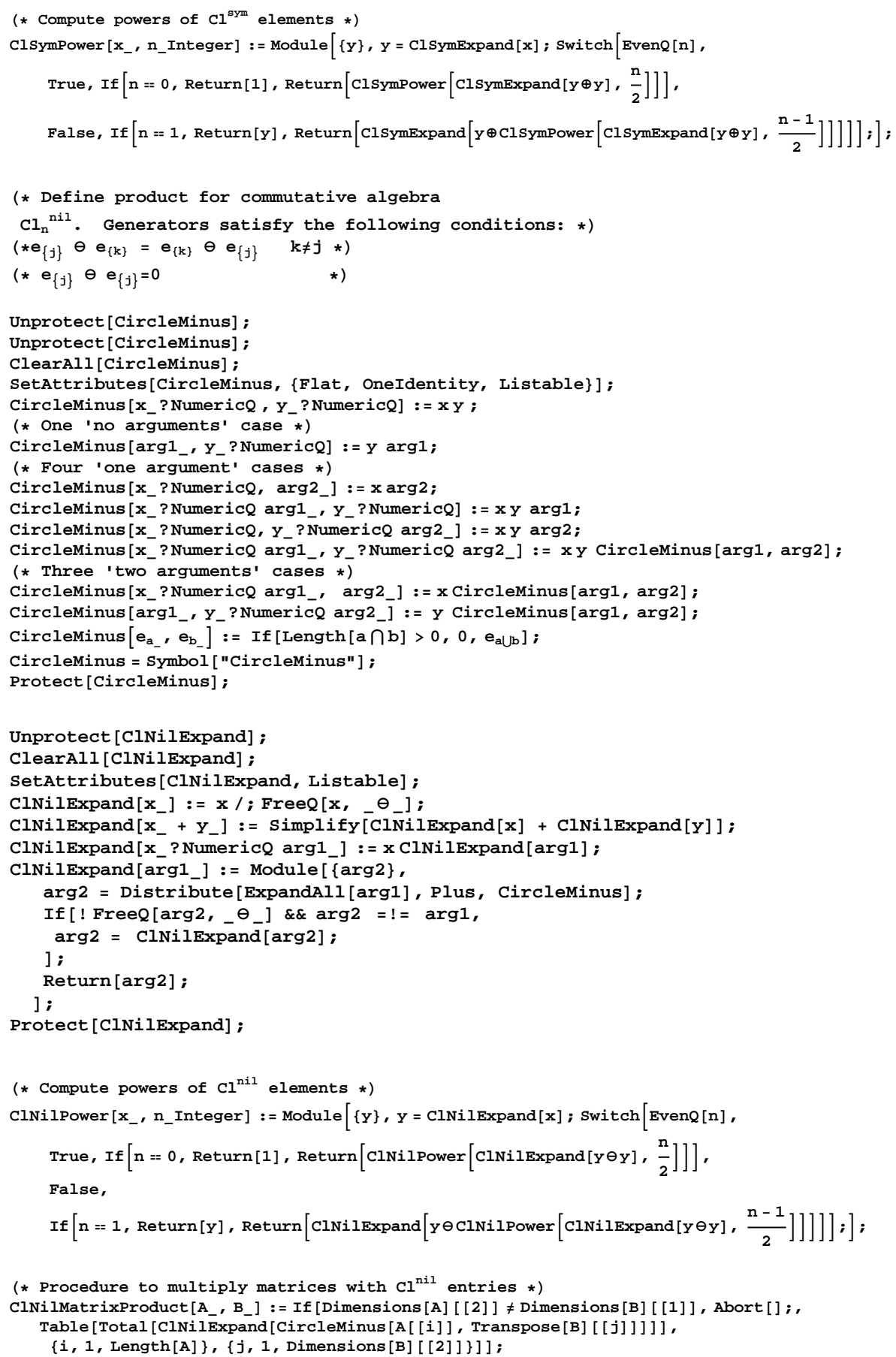




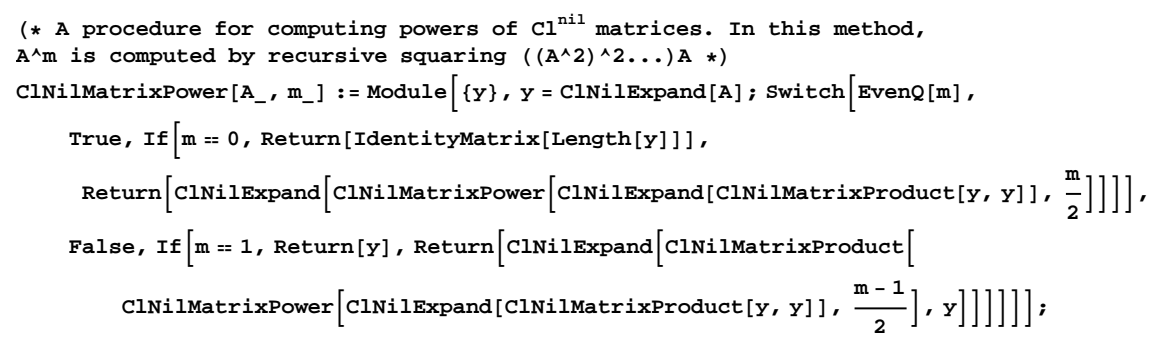

Acknowledgment. The second author thanks Marco Budinich for helpful comments and suggestions on Mathematica procedures.

\section{References}

1. C. Bettstetter, On the minimum node degree and connectivity of a wireless multihop network. Proceedings of the 3rd ACM international Symposium on Mobile Ad Hoc Networking \& Computing (Lausanne, Switzerland, June 09 - 11, 2002). MobiHoc '02. ACM, New York, NY, 2002. http://doi.acm.org/10.1145/513800.513811

2. P. Feinsilver, Zeon algebra, Fock space, and Markov chains, arXiv:0711.1680v1 [math$\mathrm{ph}]$.

3. M. Franceschetti, O. Dousse, D. N. C. Tse, P. Thiran, Closing the gap in the capacity of wireless networks via percolation theory, IEEE Transactions on Information Theory, 53 1009-1018, (2007).

4. N. Guillotin-Plantard, R. Schott, Dynamic random walks on Heisenberg groups, J. Th. Prob., 19 377-395, (2006).

5. P. Gupta, P.R. Kumar, Critical power for asymptotic connectivity in wireless networks, Stochastic analysis, control, optimization and applications: A volume in honor of W.H. Fleming, Birkhaüser, Boston, 1998.

6. P. Gupta, P.R. Kumar, The capacity of wireless networks, IEEE Transactions on Information Theory, IT-46 388-404, (2000).

7. P. Gupta, P.R.Kumar, Internets in the sky: The capacity of three dimensional wireless networks, Communications in Information and Systems, 1 33-50, (2001).

8. Z. Kong, E.M. Yeh, Characterization of the critical density for percolation in random geometric graphs, Proceedings of the 2007 International Symposium on Information Theory, Nice, France, June 24 - 29, 2007.

9. M. Penrose, Random geometric graphs, Oxford Studies in Probability, vol. 5, Oxford University Press, 2003.

10. M. Penrose, On $k$-connectivity for a geometric random graph, Random Structures and Algorithms, 15 145-164, (1999).

11. R. Schott, G.S. Staples, Random walks in Clifford algebras of arbitrary signature as walks on directed hypercubes, Markov Processes and Related Fields, to appear.

12. R. Schott, G.S. Staples, Nilpotent adjacency matrices and random graphs, Ars Combinatoria, To appear.

13. R. Schott, G.S. Staples, Reductions in computational complexity using Clifford algebras, Advances in Applied Clifford Algebras, To appear.

14. R. Schott, G.S. Staples, Connected components and evolution of random graphs: an algebraic approach, Prépublications de l'Institut Elie Cartan, No. 2007/27, (2007). 
15. R. Schott, G.S. Staples, Nilpotent adjacency matrices, random graphs, and quantum random variables, J. Phys. A: Math. Theor., 41 155205, (2008).

16. R. Schott, G.S. Staples, Cycles and components in geometric graphs: adjacency operator approach, Preprint online at http://www.siue.edu/ sstaple/index_files/ research.htm, (2008).

17. G.S. Staples, Clifford-algebraic random walks on the hypercube, Advances in Applied Clifford Algebras, 15 213-232, (2005).

18. G.S. Staples, Norms and generating functions in Clifford algebras, Advances in Applied Clifford Algebras, 18 75-92, (2008).

19. G.S. Staples, Graph-theoretic approach to stochastic integrals with Clifford algebras, J. Theor. Prob., 20 257-274, (2007).

20. F. Xue, P.R. Kumar, The number of neighbors needed for connectivity of wireless networks, Wireless Networks, 10 169-181, (2004).

René Schott

IECN and LORIA

Université Henri Poincaré-Nancy I

BP 239

54506 Vandoeuvre-lès-Nancy, France

e-mail: schott@loria.fr

G. Stacey Staples

Department of Mathematics and Statistics

Southern Illinois University Edwardsville

Edwardsville, IL 62026-1653

e-mail: sstaple@siue.edu 